TRANSACTIONS OF THE

AMERICAN MATHEMATICAL SOCIETY

Volume 352, Number 1, Pages 429-456

S 0002-9947(99)02210-2

Article electronically published on September 21, 1999

\title{
ON THE EXISTENCE OF A UNIPOTENT SUPPORT FOR THE IRREDUCIBLE CHARACTERS OF A FINITE GROUP OF LIE TYPE
}

\author{
MEINOLF GECK AND GUNTER MALLE
}

\begin{abstract}
In 1980, Lusztig posed the problem of showing the existence of a unipotent support for the irreducible characters of a finite group of Lie type. This problem was solved by Lusztig in the case where the characteristic of the field over which the group is defined is large enough. The first named author extended this to the case where the characteristic is good. It is the purpose of this paper to remove this condition as well, so that the existence of unipotent supports is established in complete generality.
\end{abstract}

\section{INTRODUCTION}

Let $G$ be a connected reductive group defined over the finite field with $q$ elements, and let $F: G \rightarrow G$ be the corresponding Frobenius map. Let $\operatorname{Irr}\left(G^{F}\right)$ be the set of (complex) irreducible characters of the finite reductive group $G^{F}$. Let $\rho \in \operatorname{Irr}\left(G^{F}\right)$ and $C$ be an $F$-stable unipotent class in $G$. Following Lusztig [12] we define an average value of $\rho$ on $C^{F}$ as follows: let $u_{1}, \ldots, u_{r} \in C^{F}$ be representatives for the $G^{F}$-classes contained in $C^{F}$. For each $j$ let $A_{G}\left(u_{j}\right)$ be the group of components of the centralizer of $u_{j}$ in $G$. Then our average value is defined as

$$
\operatorname{AV}(C, \rho):=\sum_{j=1}^{r}\left[A_{G}\left(u_{j}\right): A_{G}\left(u_{j}\right)^{F}\right] \rho\left(u_{j}\right) .
$$

It will turn out that this form of average values is more accessible to explicit computations than just averaging over the elements in $C^{F}$ themselves.

The class $C$ is called the unipotent support of $\rho$ if $C$ has maximal dimension such that $\operatorname{AV}(C, \rho) \neq 0$ and if $C$ is unique among all classes with this property.

Our main result asserts that unipotent supports always exist; furthermore, we give an explicit formula for the average value of an irreducible character on its unipotent support, see Theorem 3.7. Our method will be to reduce the case of small characteristic to that in large characteristic, which is known by Lusztig's work [12].

It has already been shown in [2, Prop. 1.3] that average values as above can be expressed in terms of the Green functions of $G^{F}$. (In fact, this is essentially the only result from [2] that we will use here.) The exact computation of these Green functions in turn involves the knowledge of the Springer correspondence for $G$. In

Received by the editors November 1, 1996 and, in revised form, July 29, 1997.

1991 Mathematics Subject Classification. Primary 20C33, 20 G40.

The second author gratefully acknowledges financial support by the Deutsche Forschungsgemeinschaft. 
good characteristic, the unipotent classes and the Springer correspondence are independent of the characteristic. In bad characteristic, the situation is rather different. We use the fact, due to Spaltenstein [19], that the partially ordered set of unipotent classes of a group in good characteristic can be embedded in that of a group of the same type but in bad characteristic. Then our main tool is a comparison result about the Springer correspondence in good and bad characteristic, see Theorem 2.1. This is derived from the explicit knowledge of the generalized Springer correspondence, as computed by Lusztig-Spaltenstein [14] and Spaltenstein [20]. This might also be of independent interest.

Our main result shows that the unipotent support of an irreducible character is 'independent of $q$ '. A precise formulation of this statement can be given using Lusztig's canonical map from irreducible characters to unipotent classes, whose definition was already given in [7] in good characteristic. Our above comparison result shows that the analogously defined map in bad characteristic shares the same formal properties as the original map in good characteristic, see Proposition 3.6.

The explicit formula for the average value of an irreducible character $\rho$ on its unipotent support $C_{\rho}$ also allows us to give 'elementary' characterizations for some invariants attached to $\rho$. Namely, in the framework of $[7,(4.23)-(4.26)]$, it is possible to give a precise sense to the statement that the degree of $\rho$ is given by a polynomial in $q$. Thus, there exist well-defined integers $n_{\rho}>0$ and $a_{\rho} \geq 0$ such that

$$
\pm n_{\rho} \rho(1)=q^{a_{\rho}}+\text { integral linear combination of higher powers of } q \text {. }
$$

The formula in Theorem 3.7 recovers $n_{\rho}$ and $a_{\rho}$ from the value $\operatorname{AV}\left(C_{\rho}, \rho\right)$, the cardinality of $A_{G}(u)$ (for $u \in C_{\rho}$ ), and the dimension of the variety of Borel subgroups containing $u$.

In Section 4, we discuss some applications of our results. We show that the notion of unipotent support behaves well with respect to twisted induction of characters from Levi subgroups and induction of unipotent classes as defined by LusztigSpaltenstein [13]. More generally, we show that there is a similar compatibility with respect to Lusztig's Jordan decomposition of characters and Spaltenstein's generalized induction of classes [19, III.12]. Furthermore, we show that the unipotent support of a cuspidal character must be a cuspidal class in the sense defined by the authors in [3]. Finally, we give some examples and extend our results to the Suzuki and Ree groups.

\section{SPRINGER CORRESPONDENCE IN GOOD AND BAD CHARACTERISTIC}

In this section we assume that $G$ is any connected reductive group defined over an algebraically closed field $k$ of characteristic $p \geq 0$. Let $W$ be the Weyl group of $G$ with respect to a fixed maximal torus $T \subseteq G$, and $S \subseteq W$ be the set of simple reflections determined by a fixed Borel subgroup $B \subseteq G$ containing $T$.

Let $X_{G}$ be the partially ordered set of unipotent classes in $G$. For each $C \in$ $X_{G}$ we fix once and for all an element $u \in C$ and let $A_{G}(u)$ be the group of components of the centralizer of $u$ in $G$. We then define $\mathcal{N}_{G}$ to be the set of all pairs $(C, \psi)$ where $C \in X_{G}$ and $\psi \in \operatorname{Irr}\left(A_{G}(u)\right.$ ) (for the chosen element $u \in C$ ). The Springer correspondence gives an injective map $i_{G}: \operatorname{Irr}(W) \rightarrow \mathcal{N}_{G}$. Note that, in the framework of [8], this works without any restriction on $G$ or $p$.

We want to compare the Springer correspondence for $G$ (or, at least, certain properties of it) with that of another group of the same type as $G$ but possibly defined over a field of characteristic different from $p$. This can be done in terms of 
results obtained by Spaltenstein [19], by relating everything to a group in characteristic 0 .

Let $G^{\prime}$ be a connected reductive group over $\mathbb{C}$ and $T^{\prime} \subseteq G^{\prime}$ be a maximal torus such that the root data of $G$ and $G^{\prime}$ with respect to $T$ and $T^{\prime}$, respectively, are isomorphic. We also fix a Borel subgroup $B^{\prime} \subseteq G^{\prime}$ containing $T^{\prime}$. Then the pair $(W, S)$ can be canonically identified with the corresponding pair in $G^{\prime}$ defined with respect to $T^{\prime} \subseteq B^{\prime}$.

Let $X_{G^{\prime}}$ be the set of unipotent classes in $G^{\prime}$. For each $C \in X_{G^{\prime}}$ we choose an element $u \in C$ and let $A_{0}(u)$ be the group of components of the centralizer of $u$ in $G^{\prime}$. We define $\mathcal{N}_{G^{\prime}}$ in an analogous way as the set $\mathcal{N}_{G}$ was defined for $G$. Then, the Springer correspondence for $G^{\prime}$ gives an injective map $i_{0}: \operatorname{Irr}(W) \rightarrow \mathcal{N}_{G^{\prime}}$.

Let $\pi_{G}: X_{G^{\prime}} \rightarrow X_{G}$ be Spaltenstein's map, see [19, Théorème III.5.2]. This map is uniquely characterized by the following three properties:

- it respects the usual partial orderings on $X_{G^{\prime}}$ and on $X_{G}$,

- it preserves the dimensions of classes, and

- it satisfies certain compatibility conditions with respect to parabolic subgroups in $G$ and $G^{\prime}$ containing $B$ and $B^{\prime}$, respectively.

Moreover, $\pi_{G}$ is an isomorphism of partially ordered sets onto its image.

If $p$ is a good prime, then $\pi_{G}$ is an isomorphism of partially ordered sets. In this case, we can also canonically identify the groups $A_{G^{\prime}}\left(u^{\prime}\right)$ and $A_{G}(u)$ (where $u^{\prime} \in C$ for some $C \in X_{G^{\prime}}$ and $u \in \pi_{G}(C)$ ), and then the Springer correspondence for $G^{\prime}$ coincides with that for $G$. This follows from the explicit results obtained by Springer, Hotta-Springer, Shoji, and Alvis-Lusztig (see [16] and the references there). If $p$ is a bad prime, the situation may be different.

Using the map $\pi_{G}$, we can now state the following result.

Theorem 2.1. Let $G^{\prime}$ be a connected reductive group over $\mathbb{C}$ and of the same type as $G$, as above. Let $i_{G}: \operatorname{Irr}(W) \rightarrow \mathcal{N}_{G}$ and $i_{0}: \operatorname{Irr}(W) \rightarrow \mathcal{N}_{G^{\prime}}$ be the Springer correspondences for $G$ and $G^{\prime}$, respectively. Let $E \in \operatorname{Irr}(W)$ and $i_{G}(E)=(C, \psi) \in$ $\mathcal{N}_{G}, i_{0}(E)=\left(C_{0}, \psi_{0}\right) \in \mathcal{N}_{G^{\prime}}$. Then the following hold.

(1) If $\psi_{0}$ is the trivial character, then $C=\pi_{G}\left(C_{0}\right)$ and $\psi$ also is the trivial character.

(2) We always have $C \leq \pi_{G}\left(C_{0}\right)$. If $p>0$ is a good prime, then $C=\pi_{G}\left(C_{0}\right)$.

(Note that the pairs in $\mathcal{N}_{G}$ of the form $(C, 1)$, where 1 stands for the trivial character, always lie in the image of $i_{G}$; see, for example, [2, Remark 3.2].)

We also consider the following, somewhat related result concerning special characters of $W$. Note that such a character always corresponds to a pair in $\mathcal{N}_{G}$ of the form $(C, 1)$ under the Springer correspondence (see [7, 13.1.1] in good characteristic and then use (1) of the above theorem for the general case).

Proposition 2.2. Let $E_{1} \in \operatorname{Irr}(W)$ be a special character and $i_{G}\left(E_{1}\right)=(C, 1) \in$ $\mathcal{N}_{G}$. If $E^{\prime} \in \operatorname{Irr}(W)$ is in the same family as $E_{1}$ and $i_{G}\left(E^{\prime}\right)=\left(C^{\prime}, \psi^{\prime}\right) \in \mathcal{N}_{G}$, then $C^{\prime} \leq C$.

By standard reduction arguments (cf. $[8,(10.1)]$ ) it suffices to prove these results in the case where $G$ is simple. Note also that the Springer correspondence is insensitive to the isogeny type of $G$. Taking into account the remarks preceding Theorem 2.1 we see that, in order to prove this theorem, we only need to consider a group $G$ over a field of bad characteristic $p$. Once this is established it suffices to prove Proposition 2.2 only for a group $G^{\prime}$ of the same type as $G$ but over $\mathbb{C}$. 
If $G$ is of type $A_{n}$, then all primes are good and all characters of $W$ are special. Hence we are done in this case. If $G$ is of exceptional type $G_{2}, F_{4}, E_{6}, E_{7}$ or $E_{8}$, the statements in Theorem 2.1 and Proposition 2.2 are easily read off the tables in $[20,5.4]$, and those in [7, Chap. 4] concerning families of characters.

In the remainder of this section we will consider a group $G$ of classical type $B_{n}$, $C_{n}$ or $D_{n}$ over a field of characteristic 2 . As before let $G^{\prime}$ be the corresponding group of the same type over $\mathbb{C}$. The statements in Theorem 2.1 and Proposition 2.2 will follow by comparing several combinatorial maps defined in [8], [14], [19]. More precisely, we describe combinatorially the map $i_{0}: \operatorname{Irr}(W) \rightarrow \mathcal{N}_{G^{\prime}}$ for $G^{\prime}$ and the map $i_{G}: \operatorname{Irr}(W) \rightarrow \mathcal{N}_{G}$ for $G$. Furthermore, let $p_{0}: \mathcal{N}_{G^{\prime}} \rightarrow X_{G^{\prime}}$ and $p_{G}: \mathcal{N}_{G} \rightarrow$ $X_{G}$ denote the projection maps onto the first factor. These have natural sections $s_{0}: X_{G^{\prime}} \rightarrow \mathcal{N}_{G^{\prime}}$ and $s_{G}: X_{G} \rightarrow \mathcal{N}_{G}$ defined by $C \mapsto(C, 1)$. We will sometimes identify elements of $X$ with their image in $\mathcal{N}$. We have to study the resulting diagram of maps

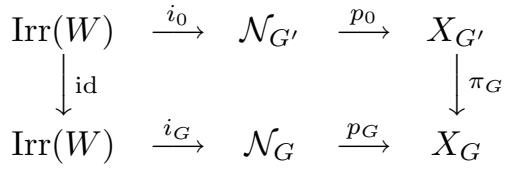

A. Some combinatorics. In order to write down the Springer correspondence and the map $\pi_{G}$ for groups of classical type we first have to recall the definition of certain combinatorial objects, so-called generalized symbols. These were first introduced by Lusztig and Spaltenstein.

Let $n, r, s$ be positive integers and $d \in\{0,1\}$. Following [14, $\S 1]$ we define $\tilde{X}_{n, d}^{r, s}$ to be the set of ordered pairs $(A, B)$ of finite sequences $A=\left(a_{1}, \ldots, a_{m+d}\right)$, $B=\left(b_{1}, \ldots, b_{m}\right)$ with

$$
\begin{aligned}
& \text { (1) } a_{i}-a_{i-1} \geq r+s \text { for } 1<i \leq m+d, \\
& \text { (2) } b_{i}-b_{i-1} \geq r+s \text { for } 1<i \leq m, \\
& \text { (3) } b_{1} \geq s, \\
& \text { (4) } \sum_{i=1}^{m+d} a_{i}+\sum_{i=1}^{m} b_{i}=n+r m(m+d-1)+\operatorname{sm}(m+d) \text {. }
\end{aligned}
$$

On $\tilde{X}_{n, d}^{r, s}$ there is a shift operation $\tilde{X}_{n, d}^{r, s} \rightarrow \tilde{X}_{n, d}^{r, s}$ defined by

$$
(A, B) \mapsto\left(\left(0, a_{1}+r+s, \ldots, a_{m+d}+r+s\right),\left(s, b_{1}+r+s, \ldots, b_{m}+r+s\right)\right) .
$$

The quotient of $\tilde{X}_{n, d}^{r, s}$ by this shift is denoted by $X_{n, d}^{r, s}$; its elements are called symbols and will also be denoted by representatives $(A, B)$. The set $X_{0, d}^{r, s}$ consists of a single element $\Lambda_{0, d}^{r, s}$ : if $d=0$, then $\Lambda_{0,0}^{r, s}:=(-,-)$; if $d=1$, then $\Lambda_{0,1}^{r, s}:=((0),-)$. There exists an addition

$$
X_{n, d}^{r, s} \times X_{n^{\prime}, d}^{r^{\prime}, s^{\prime}} \longrightarrow X_{n+n^{\prime}, d}^{r+r^{\prime}, s+s^{\prime}}
$$

defined by component-wise addition of representatives of the same length. An element $\Lambda=(A, B) \in X_{n, d}^{r, s}$ is called distinguished if $a_{1} \leq b_{1} \leq a_{2} \leq \ldots \leq b_{m}$ (and $b_{m} \leq a_{m+1}$ if $\left.d=1\right)$. The subset of $X_{n, d}^{r, s}$ of distinguished symbols is denoted by $D_{n, d}^{r, s}$. Two (classes of) symbols are called similar if the multisets of the entries of suitable representatives coincide. Thus for any symbol $\Lambda \in X_{n, d}^{r, s}$ there exists a unique distinguished symbol $D(\Lambda)$ in $X_{n, d}^{r, s}$ which is similar to $\Lambda$. 
Finally, if $s=0$, we denote by $Y_{n, 0}^{r}$ the quotient of $X_{n, 0}^{r, 0}$ by the operation of swapping the two rows of a symbol, and we write $Y_{n, 1}^{r}$ for $X_{n, 1}^{r, 0}$.

We introduce a sorting procedure $R^{r, s}: X_{n, d}^{r, s} \rightarrow X_{n, d}^{r, s}$ on symbols $\Lambda \in X_{n, d}^{r, s}$ as follows. If $\Lambda=(A, B)$, write

$$
\left(c_{1}, c_{2}, c_{3}, \ldots\right):=\left(a_{1}, b_{1}, a_{2}, b_{2}, \ldots\right) .
$$

If $c_{i} \leq c_{i+1}$ for all $i$, then $R^{r, s}(\Lambda):=\Lambda$. Otherwise, let $j$ be the minimal index with $c_{j}>c_{j+1}$, and let $l$ be maximal such that $c_{j+2 k}>c_{j+2 k+1}$ for all $0 \leq k \leq l$. Define $R^{r, s}(\Lambda):=\left(\tilde{c}_{1}, \tilde{c}_{2}, \ldots\right)$ where

$$
\tilde{c}_{i}:= \begin{cases}c_{i+1} & \text { if } i=j+2 k, 0 \leq k \leq l, \\ c_{i-1} & \text { if } i=j+2 k+1,0 \leq k \leq l, \\ c_{i} & \text { otherwise. }\end{cases}
$$

Since $\Lambda \in X_{n, d}^{r, s}$, we have $c_{i} \leq c_{i+2}-r-s$ for all $i$. Now

$$
\begin{aligned}
& \tilde{c}_{j}=c_{j+1} \geq c_{j-1}+r+s \geq c_{j-2}+r+s=\tilde{c}_{j-2}+r+s, \\
& \tilde{c}_{j+1}=c_{j}>c_{j+1} \geq c_{j-1}+r+s=\tilde{c}_{j-1}+r+s,
\end{aligned}
$$

by the choice of $j$, and

$$
\begin{aligned}
& \tilde{c}_{j+2 l}=c_{j+2 l+1}<c_{j+2 l} \leq c_{j+2 l+2}-r-s=\tilde{c}_{j+2 l+2}-r-s, \\
& \tilde{c}_{j+2 l+1}=c_{j+2 l} \leq c_{j+2 l+2}-r-s \leq c_{j+2 l+3}-r-s=\tilde{c}_{j+2 l+3}-r-s,
\end{aligned}
$$

by the choice of $l$, so $R^{r, s}(\Lambda) \in X_{n, d}^{r, s}$ as well, and it is similar to $\Lambda$. Moreover the smallest index $i$ such that $\tilde{c}_{i}>\tilde{c}_{i+1}$ is strictly larger than $j$. Thus there exists an $m \geq 0$ such that $\left(R^{r, s}\right)^{m}(\Lambda) \in D_{n, d}^{r, s}$ is distinguished. We also write $\left(R^{r, s}\right)^{\infty}(\Lambda)$ for this distinguished symbol $\left(R^{r, s}\right)^{m}(\Lambda)$.

For $\Lambda^{\prime} \in X_{n, d}^{r+r^{\prime}, s+s^{\prime}}$ we define $R^{r, s}\left(\Lambda^{\prime}\right):=R^{r, s}\left(\Lambda^{\prime}-\Lambda_{0, d}^{r^{\prime}, s^{\prime}}\right)+\Lambda_{0, d}^{r^{\prime}, s^{\prime}}$, so its entries $\left(\tilde{c}_{1}^{\prime}, \tilde{c}_{2}^{\prime}, \ldots\right)$ are obtained from the entries $\left(c_{1}^{\prime}, c_{2}^{\prime}, \ldots\right)$ of $\Lambda^{\prime}$ via

$$
\begin{array}{ll}
\tilde{c}_{j+2 k}^{\prime}=c_{j+2 k+1}^{\prime}-s^{\prime}, & \tilde{c}_{j+2 k+1}^{\prime}=c_{j+2 k}^{\prime}+s^{\prime} \quad \text { if } j \text { is odd, } \\
\tilde{c}_{j+2 k}^{\prime}=c_{j+2 k+1}^{\prime}-r^{\prime}, & \tilde{c}_{j+2 k+1}^{\prime}=c_{j+2 k}^{\prime}+r^{\prime}
\end{array}
$$

for $0 \leq k \leq l$, and $\tilde{c}_{i}^{\prime}=c_{i}^{\prime}$ otherwise. Clearly, both operations carry over to $Y_{n, d}^{r}$ and $Y_{n, d}^{r+r^{\prime}}$.

B. The symplectic groups. Assume that $G, G^{\prime}$ are of adjoint type $C_{n}$. Let $W_{n}$ be the Weyl group of $G$ of type $C_{n}$. The irreducible characters of $W_{n}$ can be parametrized by pairs of partitions $(\alpha, \beta)$ of $n$, i.e., $\alpha=\left(\alpha_{1} \leq \alpha_{2} \leq \ldots \leq \alpha_{m^{\prime}}\right)$, $\beta=\left(\beta_{1} \leq \beta_{2} \leq \ldots \leq \beta_{m}\right)$ with $\sum \alpha_{i}+\sum \beta_{i}=n$. By adding zeros we may normalize $(\alpha, \beta)$ so that $m^{\prime}=m+1$, so this yields a natural parametrization

$$
\operatorname{Irr}\left(W_{n}\right) \stackrel{\sim}{\longrightarrow} X_{n, 1}^{0,0}, \quad(\alpha, \beta) \mapsto(\alpha, \beta) .
$$

Via the addition defined above we also get bijections

$$
\psi_{G^{\prime}}: \operatorname{Irr}\left(W_{n}\right) \cong X_{n, 1}^{0,0} \stackrel{\sim}{\longrightarrow} X_{n, 1}^{1,1}, \quad(\alpha, \beta) \mapsto(\alpha, \beta)+\Lambda_{0,1}^{1,1},
$$

and

$$
\psi_{G}: \operatorname{Irr}\left(W_{n}\right) \cong X_{n, 1}^{0,0} \stackrel{\sim}{\longrightarrow} X_{n, 1}^{2,2}, \quad(\alpha, \beta) \mapsto(\alpha, \beta)+\Lambda_{0,1}^{2,2} .
$$

So in particular

$$
\psi_{G}((\alpha, \beta))=\psi_{G^{\prime}}((\alpha, \beta))+\Lambda_{0,1}^{1,1}
$$


We will henceforth replace $\operatorname{Irr}\left(W_{n}\right)$ in the above diagram (2.3) by $X_{n, 1}^{1,1}$ respectively $X_{n, 1}^{2,2}$ (see also $[8,12.2 .4]$ and $\left.[14,1.3]\right)$.

We now define the inverse of $i_{0}$ on $X_{G^{\prime}} \subset \mathcal{N}_{G^{\prime}}$. The unipotent classes of $G^{\prime}$ are parametrized by partitions $\lambda$ of $2 n$ such that any odd part occurs an even number of times. Let $\lambda$ be such a partition. By adding zeros we may arrange so that $\lambda_{1}=0$ and the number of parts of $\lambda$ is odd. It can easily be checked that the map described in $[8,11.6]$ can be given as follows. Partition the sequence $\left(\lambda_{1}, \lambda_{2}, \ldots, \lambda_{r}\right)$ into blocks of lengths one or two such that all even $\lambda_{i}$ lie in a block of length 1 and the odd $\lambda_{i}$ lie in blocks of length 2 . Define a sequence $\left(c_{1}^{\prime}, \ldots, c_{r}^{\prime}\right)$ by

$$
\left.\begin{array}{l}
c_{i}^{\prime}:=\lambda_{i} / 2+(i-1) \quad \text { if }\left\{\lambda_{i}\right\} \text { is a block, } \\
c_{i}^{\prime}:=\left(\lambda_{i}+1\right) / 2+i-1, \\
\left.c_{i+1}^{\prime}:=\left(\lambda_{i+1}+1\right) / 2+i-1=c_{i}^{\prime}\right\}
\end{array}\right\} \quad \text { if }\left\{\lambda_{i}, \lambda_{i+1}\right\} \text { lie in one block. }
$$

Then by $\left[8\right.$, Thm. 12.3] $i_{0}^{-1}((\lambda, 1))$ is the unique distinguished symbol in $X_{n, 1}^{1,1}$ with entries $c_{1}^{\prime}, \ldots, c_{r}^{\prime}$. Conversely, it can easily be seen that every distinguished symbol can be obtained this way, so $i_{0}$ restricts to a bijection $i_{0}: D_{n, 1}^{1,1} \stackrel{\sim}{\rightarrow} X_{G^{\prime}}$. Furthermore, the image $i_{0}^{-1}((\lambda, \psi))$ for arbitrary $\psi$ is a symbol similar to $i_{0}^{-1}((\lambda, 1))$.

The unipotent classes of $G$ are parametrized by pairs $(\lambda, \epsilon)$ where $\lambda$ is as before and $\epsilon:\left\{\lambda_{1}, \ldots, \lambda_{r}\right\} \rightarrow\{0,1, \omega\}$ is a certain sequence of signs, such that $\epsilon_{\lambda_{i}}=\omega$ on parts $\lambda_{i}$ of odd length (see [19, I.2.5, I.2.6]). The generalized Springer correspondence $i_{G}$ is described in $[14,2.1]$. Let $(\lambda, \epsilon)$ be the label of a unipotent class. Assume again that $\lambda_{1}=0$ and the number $r$ of parts of $\lambda$ is odd. Furthermore, partition the parts of $\lambda$ into blocks of lengths 1 or 2 such that $\lambda_{i}$ lies in a block of length 1 if and only if $\epsilon_{\lambda_{i}}=1$. Then integers $c_{i}$ are defined as follows:

$$
\begin{aligned}
& c_{i}:=\lambda_{i} / 2+2(i-1) \quad \text { if }\left\{\lambda_{i}\right\} \text { is a block, } \\
& \left.\begin{array}{l}
c_{i}:=\left(\lambda_{i}+1\right) / 2+2(i-1), \\
c_{i+1}:=\left(\lambda_{i}-1\right) / 2+2 i=c_{i}+1
\end{array}\right\} \quad \text { if }\left\{\lambda_{i}, \lambda_{i+1}\right\} \text { is a block, } \epsilon_{\lambda_{i}} \neq 0, \\
& \left.\begin{array}{l}
c_{i}:=\left(\lambda_{i}+2\right) / 2+2(i-1), \\
c_{i+1}:=\left(\lambda_{i}-2\right) / 2+2 i=c_{i}
\end{array}\right\} \quad \text { if }\left\{\lambda_{i}, \lambda_{i+1}\right\} \text { is a block, } \epsilon_{\lambda_{i}}=0 .
\end{aligned}
$$

The image of $(\lambda, \epsilon)$ under $i_{G}^{-1}$ is then the unique distinguished symbol with entries $c_{1}, \ldots, c_{r}$. Thus again $i_{G}$ restricts to a bijection $i_{G}: D_{n, 1}^{2,2} \stackrel{\sim}{\rightarrow} X_{G}$.

Finally, according to [19, III.6.1] the map $\pi_{G}$ is given by

$$
\pi_{G}: \mathcal{N}_{G^{\prime}} \longrightarrow \mathcal{N}_{G}, \quad \lambda \mapsto\left(\lambda, \epsilon_{1}\right),
$$

where $\epsilon_{1}$ is defined by the property that no entry of $\epsilon_{1}$ is equal to 0 ; in particular $\left(\lambda, \epsilon_{1}\right)$ is the class of largest dimension among all classes parametrized by pairs $(\lambda, \epsilon)$ (loc. cit.).

We have now given combinatorial descriptions for all maps in (2.3).

Proposition 2.8. Theorem 2.1 and Proposition 2.2 hold for groups of type $\mathrm{Sp}_{2 n}$.

Proof. By what we introduced above, Theorem 2.1(1) is equivalent to the statement that

$$
\pi_{G} \circ i_{0}=i_{G} \circ \pi_{1,1}^{2,2} \quad \text { on } D_{n, 1}^{1,1},
$$


where $\pi_{1,1}^{2,2}: D_{n, 1}^{1,1} \rightarrow D_{n, 1}^{2,2}$ is the bijection $\Lambda \mapsto \Lambda+\Lambda_{0,1}^{1,1}$. But by (2.5) this amounts to showing $c_{i}=c_{i}^{\prime}+(i-1)$ for the integers defined in (2.6) and (2.7), which is clear. (Note that the third case in (2.7) can not occur by the definition of $\pi_{G}$.)

For part (2) of Theorem 2.1 let $E \in \operatorname{Irr}\left(W_{n}\right)$ and $c_{1}, c_{2}, \ldots, c_{r}$ be the entries of $\Lambda:=\psi_{G}(E) \in X_{n, 1}^{2,2}$. Then the entries of $\Lambda^{\prime}:=\psi_{G^{\prime}}(E) \in X_{n, 1}^{1,1}$ are $\left(c_{1}, c_{2}-\right.$ $\left.1, \ldots, c_{r}-r+1\right)$. We apply the sorting procedure $R^{1,1}$. Namely, $R^{1,1}\left(\Lambda^{\prime}\right)$ is similar to $\Lambda^{\prime}$; thus the unipotent class corresponding to it under the generalized Springer map $i_{0}$ remains the same. On the other hand, the class $\tilde{C}:=i_{G}\left(R^{1,1}(\Lambda)\right)$ is larger than the class $C:=i_{G}(\Lambda)$ by the description of the partial order on $X_{G}$ in $[19$, I.2.10]: if we had $c_{j}=c_{j+1}$, then the label for $\tilde{C}$ is obtained from the one for $C$ by changing $\epsilon_{\lambda_{i}}$ from 0 to 1 , and otherwise the corresponding partition is replaced by a strictly smaller one. Thus we have

$$
i_{0}\left(\left(R^{1,1}\right)^{\infty}\left(\Lambda^{\prime}\right)\right)=i_{0}\left(\Lambda^{\prime}\right), \quad i_{G}\left(\left(R^{1,1}\right)^{\infty}(\Lambda)\right) \geq i_{G}(\Lambda),
$$

and $\pi_{G}\left(i_{0}\left(\left(R^{1,1}\right)^{\infty}\left(\Lambda^{\prime}\right)\right)\right)=i_{G}\left(\left(R^{1,1}\right)^{\infty}(\Lambda)\right)$ by the first part, so $i_{G}(\Lambda) \leq \pi_{G}\left(i_{0}\left(\Lambda^{\prime}\right)\right)$, which proves Theorem 2.1(2).

We now prove Proposition 2.2 (for $\left.G^{\prime}\right)$. Now let $E^{\prime}=(\alpha, \beta) \in \operatorname{Irr}\left(W_{n}\right)$ and $\Lambda^{\prime}:=(\alpha, \beta)+\Lambda_{0,1}^{1,0} \in X_{n, 1}^{1,0}$ be the corresponding sequences of $\beta$-numbers. By [7, $4.5]$ it is known that $E^{\prime}$ is special if and only if $\Lambda^{\prime}$ is distinguished, and moreover two characters in the same family have similar symbols. Thus $\left(R^{1,0}\right)^{\infty}\left(\Lambda^{\prime}\right)$ is the symbol for the special character in the family of $E^{\prime}$. Let $\Lambda=\psi_{G^{\prime}}\left(E^{\prime}\right)=(\alpha, \beta)+\Lambda_{0,1}^{1,1} \in X_{n, 1}^{1,1}$ be the symbol for the Springer correspondent of $E^{\prime}$. It is easily checked using $\left[19\right.$, I.2.10] that $i_{0}\left(R^{1,0}(\Lambda)\right) \geq i_{0}(\Lambda)$ in the partial order relation. Thus repeated application of $R^{1,0}$ gives the assertion of Proposition 2.2.

Remark. It was pointed out to us by Frank Lübeck that the description of the generalized Springer correspondence in [8], [14] for $G$ a symplectic group is not quite correct. Nevertheless, according to Shoji it remains true that the images of $(u, \psi),\left(u^{\prime}, \psi^{\prime}\right)$ are similar symbols if and only if $u=u^{\prime}$. Moreover the image of $(u, 1)$ is as described above (see [18, Remark 5.8] for the case of odd characteristic). These are the only facts we need in our argument.

C. The odd-dimensional orthogonal groups. Assume now that $G, G^{\prime}$ are of adjoint type $B_{n}$. Then there exists a bijective isogeny from $G$ to a simple group of adjoint type $C_{n}$. To check Theorem 2.1 for $\mathrm{SO}_{2 n+1}$ we will compare the classes and the Springer correspondence in good characteristic with those of $\mathrm{Sp}_{2 n}$ in characteristic 2 . As above, let $W_{n}$ be the Weyl group of type $C_{n}$. We have to describe the maps in diagram (2.3). First, $\operatorname{Irr}\left(W_{n}\right)$ is identified with $Y_{n, 1}^{2}$ via

$$
\psi_{G^{\prime}}: \operatorname{Irr}\left(W_{n}\right) \cong X_{n, 1}^{0,0} \stackrel{\sim}{\longrightarrow} Y_{n, 1}^{2}, \quad(\alpha, \beta) \mapsto(\alpha, \beta)+\Lambda_{0,1}^{2,0},
$$

where again $(\alpha, \beta)$ is normalized such that $\alpha$ has one part more than $\beta$. We will henceforth replace $\operatorname{Irr}\left(W_{n}\right)$ by $Y_{n, 1}^{2}$ (respectively $X_{n, 1}^{2,2}$ via $\left.(2.4)\right)$.

The unipotent classes of $G^{\prime}$ are parametrized by partitions $\lambda$ of $2 n+1$ such that any even part occurs an even number of times. Let $\lambda$ be such a partition. Note that $\lambda$ has an odd number of parts. It can easily be checked that the map described in $[8,11.7]$ can be given as follows. Partition the sequence $\left(\lambda_{1}, \lambda_{2}, \ldots, \lambda_{r}\right)$ into blocks of lengths one or two such that all odd $\lambda_{i}$ lie in a block of length 1 and the even $\lambda_{i}$ 
lie in blocks of length 2. Then define

$$
\left.\begin{array}{l}
c_{i}^{\prime}:=\left(\lambda_{i}-1\right) / 2+i-1 \quad \text { if }\left\{\lambda_{i}\right\} \text { is a block, } \\
c_{i}^{\prime}:=\lambda_{i} / 2+i-1, \\
c_{i+1}^{\prime}:=\lambda_{i+1} / 2+i-1=c_{i}^{\prime}
\end{array}\right\} \quad \text { if }\left\{\lambda_{i}, \lambda_{i+1}\right\} \text { lie in one block. }
$$

Then by $\left[8\right.$, Thm. 13.3] $i_{0}^{-1}((\lambda, 1))$ is the unique distinguished symbol in $Y_{n, 1}^{2}$ with entries $c_{1}^{\prime}, \ldots, c_{r}^{\prime}$. It is easily seen that $i_{0}$ restricts to a bijection $i_{0}: D_{n, 1}^{2} \stackrel{\sim}{\rightarrow} X_{G^{\prime}}$. Furthermore, $i_{0}^{-1}((\lambda, \psi))$ is similar to $i_{0}^{-1}((\lambda, 1))$ for any $\psi$.

We have already given the generalized Springer correspondence for $\mathrm{Sp}_{2 n}$ in Section B. It remains to describe the map $\pi_{G}$ from the partially ordered set of unipotent classes $X_{G^{\prime}}$ of $G^{\prime}$ to the partially ordered set of unipotent classes of $\mathrm{Sp}_{2 n}$ in characteristic 2. Let $\lambda^{\prime}$ be the label of a unipotent class of $G^{\prime}$. Then $\pi_{G}\left(\lambda^{\prime}\right)$ has the label $(\lambda, \epsilon)$ where

$$
\lambda_{i}:= \begin{cases}\lambda_{i}^{\prime}-1 & \text { if } \lambda_{i}^{\prime} \text { and } i \text { are odd, } \lambda_{i-1}^{\prime}<\lambda_{i}^{\prime} \\ \lambda_{i}^{\prime}+1 & \text { if } \lambda_{i}^{\prime} \text { is odd, } i \text { is even, } \lambda_{i}^{\prime}<\lambda_{i+1}^{\prime} \\ \lambda_{i}^{\prime} & \text { otherwise, }\end{cases}
$$

and $\epsilon_{\lambda_{i}}=0$ if and only if both $\lambda_{i}^{\prime}$ and $i$ are even (see [19, III.8.2]). We have now given combinatorial descriptions for all maps in (2.3).

Proposition 2.12. Theorem 2.1 and Proposition 2.2 hold for type $\mathrm{SO}_{2 n+1}$.

Proof. We first consider the statements in Theorem 2.1. Note that if $\psi_{G^{\prime}}(E)$ is distinguished for some $E \in \operatorname{Irr}\left(W_{n}\right)$, then also $\psi_{G}(E)$ is distinguished by (2.4) and (2.9). Let $\left(c_{1}^{\prime}, \ldots, c_{r}^{\prime}\right)$ be the sequence of entries of the distinguished symbol $\psi_{G^{\prime}}(E)$, where $E$ is labeled by $(\alpha, \beta)$. We subdivide the $c_{i}^{\prime}$ into blocks of lengths 1 or 2 , such that $\left\{c_{i}^{\prime}\right\}$ is a block if and only if $c_{i}^{\prime} \neq c_{i-1}^{\prime}, c_{i}^{\prime} \neq c_{i+1}^{\prime}$, and $c_{i}^{\prime} \neq c_{i+1}^{\prime}-1$ if $i$ is even. Then by (2.10) the parts of the partition $\lambda^{\prime}=i_{0}(E)$ are seen to be given by the formula

$$
\begin{aligned}
& \lambda_{i}^{\prime}:=2 \alpha_{k}+1 \quad \text { if } i=2 k-1, \beta_{k-1}<\alpha_{k}<\beta_{k}, \\
& \lambda_{i}^{\prime}:=2 \beta_{k}-1 \quad \text { if } i=2 k, \alpha_{k}<\beta_{k}<\alpha_{k+1}+1 \text {, } \\
& \left.\begin{array}{l}
\lambda_{i}^{\prime}:=2 \alpha_{k}, \\
\lambda_{i+1}^{\prime}:=2 \beta_{k}=\lambda_{i}^{\prime}
\end{array}\right\} \quad \text { if } i=2 k-1, \beta_{k}=\alpha_{k}, \\
& \left.\begin{array}{l}
\lambda_{i}^{\prime}:=2 \beta_{k}-1, \\
\lambda_{i+1}^{\prime}:=2 \alpha_{k+1}+1=\lambda_{i}^{\prime}
\end{array}\right\} \quad \text { if } i=2 k, \beta_{k}=\alpha_{k+1}+1, \\
& \left.\begin{array}{l}
\lambda_{i}^{\prime}:=2 \beta_{k}-2, \\
\lambda_{i+1}^{\prime}:=2 \alpha_{k+1}+2=\lambda_{i}^{\prime}
\end{array}\right\} \quad \text { if } i=2 k, \beta_{k}=\alpha_{k+1}+2 .
\end{aligned}
$$

(The fact that $\psi_{G^{\prime}}(E)$ is distinguished shows that the above covers all cases that can arise.) On the other hand, the generalized Springer correspondence for $\mathrm{Sp}_{2 n}$ 
described in (2.7) yields

$$
\begin{aligned}
& \lambda_{i}:=2 \alpha_{k} \quad \text { if } i=2 k-1, \beta_{k-1}<\alpha_{k}<\beta_{k}, \\
& \left.\begin{array}{l}
\lambda_{i}:=2 \beta_{k} \\
\lambda_{i}:=2 \alpha_{k}, \\
\lambda_{i+1}:=2 \beta_{k}=\lambda_{i}
\end{array}\right\} \quad \text { if } i=2 k, \alpha_{k}<\beta_{k}<\alpha_{k+1}+1, \\
& \left.\begin{array}{l}
\lambda_{i}:=2 \beta_{k}-1, \\
\lambda_{i+1}:=2 \alpha_{k+1}+1=\lambda_{i}
\end{array}\right\} \quad \text { if } i=2 k, \beta_{k}=\alpha_{k+1}+1, \\
& \left.\begin{array}{l}
\lambda_{i}:=2 \beta_{k}-2, \\
\lambda_{i+1}:=2 \alpha_{k+1}+2=\lambda_{i}
\end{array}\right\} \quad \text { if } i=2 k, \beta_{k}=\alpha_{k+1}+2,
\end{aligned}
$$

and $\epsilon_{\lambda_{i}}=0$ only in the last case, for the image $(\lambda, \epsilon)=i_{G}(E)$. A comparison with (2.11) now shows that $\lambda$ in (2.14) can be obtained from $\lambda^{\prime}$ in (2.13) via $\pi_{G}$, which is assertion (1).

For (2) let $E \in \operatorname{Irr}\left(W_{n}\right)$ with label $(\alpha, \beta)$. Similar to the proof of Proposition 2.8 we apply $R^{2,0}$ to change $E$ in such a way that the class $i_{0}(E)$ remains the same, and the class of $i_{G}(E)$ increases. It only remains to check that $i_{G}\left(R^{2,0}(\Lambda)\right) \geq i_{G}(\Lambda)$ for $\Lambda:=\psi_{G}(E)$, which follows from $(2.14)$ by [19, I.2.10]. This proves (2), and hence completes the proof of Theorem 2.1.

Proposition 2.2 is proved precisely as in the case of $\mathrm{Sp}_{2 n}$ using the map $R^{1,0}$ and the characterization of families and special characters in $[7,4.5]$.

D. The even-dimensional orthogonal groups. Assume that $G, G^{\prime}$ are of type $\mathrm{SO}_{2 n}$. We write $\hat{G}, \hat{G}^{\prime}$ for the extension of $G, G^{\prime}$ by the graph automorphism of order 2, i.e., for the general orthogonal group $\mathrm{GO}_{2 n}$. In [8], [14] the generalized Springer correspondence is only given for orbits under this graph-automorphism. So here let $\mathcal{N}_{G^{\prime}}, \mathcal{N}_{G}$ be the set of pairs $(C, \psi)$ consisting of a unipotent element of $G^{\prime}$ (resp. $G$ ) and a character of the group of connected components of its centralizer, up to conjugation in $\hat{G}^{\prime}, \hat{G}$, and $X_{G^{\prime}}, X_{G}$ the partially ordered sets of unipotent conjugacy classes of $\hat{G}^{\prime}, \hat{G}$ contained in $G^{\prime}, G$ respectively.

Let $W_{n}^{\prime}$ be the Weyl group of type $D_{n}$ considered as a subgroup of $W_{n}, \operatorname{Irr}\left(W_{n}\right)^{\prime}$ the set of orbits of irreducible characters of $W_{n}^{\prime}$ under the action of $W_{n}$. Then $\operatorname{Irr}\left(W_{n}\right)^{\prime}$ is parametrized by unordered pairs of partitions $(\alpha, \beta)$ of $n$. Following [8, 13.2.5] we first identify $\operatorname{Irr}\left(W_{n}\right)^{\prime}$ with $Y_{n, 0}^{2}$ via

$$
\psi_{G^{\prime}}: \operatorname{Irr}\left(W_{n}\right)^{\prime} \cong Y_{n, 0}^{0} \stackrel{\sim}{\longrightarrow} Y_{n, 0}^{2}, \quad(\alpha, \beta) \mapsto(\alpha, \beta)+\Lambda_{0,0}^{2,0},
$$

where $(\alpha, \beta)$ is normalized such that $\alpha$ and $\beta$ have the same number of parts. In characteristic 2 , we define

$$
\psi_{G}: \operatorname{Irr}\left(W_{n}\right)^{\prime} \cong Y_{n, 0}^{0} \stackrel{\sim}{\longrightarrow} Y_{n, 0}^{4}, \quad(\alpha, \beta) \mapsto(\alpha, \beta)+\Lambda_{0,0}^{4,0},
$$

where $(\alpha, \beta)$ is normalized as before (see $[14,3.3])$. We will henceforth replace $\operatorname{Irr}\left(W_{n}\right)^{\prime}$ by $Y_{n, 0}^{2}$, respectively $Y_{n, 0}^{4}$.

The unipotent classes of $\hat{G}^{\prime}$ contained in $G^{\prime}$ are parametrized by partitions $\lambda$ of $2 n$ such that any even part occurs an even number of times [19, I.2.5]. Let $\lambda$ be such a partition. Note that $\lambda$ has an even number of parts. Partition the sequence $\left(\lambda_{1}, \lambda_{2}, \ldots, \lambda_{r}\right)$ into blocks of lengths one or two such that all odd $\lambda_{i}$ lie in a block of length 1 and the even $\lambda_{i}$ lie in blocks of length 2 . It can easily be checked that the map described in $[8,11.7]$ is again given by $(2.10)$. Thus by $[8$, Thm. 13.3] 
$i_{0}^{-1}((\lambda, 1))$ is the unique distinguished symbol in $Y_{n, 0}^{2}$ with entries $c_{1}^{\prime}, \ldots, c_{r}^{\prime}$. So $i_{0}$ restricts to a bijection $i_{0}: D_{n, 0}^{2} \stackrel{\sim}{\rightarrow} X_{G^{\prime}}$.

The unipotent classes of $\hat{G}$ contained in $G$ are parametrized by pairs $(\lambda, \epsilon)$ where $\lambda$ is a partition of $2 n$ in which every odd part occurs an even number of times, and $\epsilon:\left\{\lambda_{1}, \ldots, \lambda_{r}\right\} \rightarrow\{0,1, \omega\}$ is a certain sequence of signs, which is equal to $\omega$ on parts of odd length (see $[19$, I.2.6]). The generalized Springer correspondence is described in $[14,3.1]$. Let $(\lambda, \epsilon)$ be the parameter of a unipotent class. Assume that the number $r$ of parts of $\lambda$ is even. Furthermore, partition the entries of $\lambda$ into blocks of lengths 1 or 2 such that $\lambda_{i}$ lies in a block of length 1 if and only if $\epsilon_{\lambda_{i}}=1$. Then integers $c_{i}$ are defined as follows:

$$
\begin{aligned}
& c_{i}:=\left(\lambda_{i}-2\right) / 2+2(i-1) \quad \text { if }\left\{\lambda_{i}\right\} \text { is a block, } \\
& \left.\begin{array}{l}
c_{i}:=\left(\lambda_{i}-1\right) / 2+2(i-1), \\
c_{i+1}:=\left(\lambda_{i}-3\right) / 2+2 i=c_{i}+1
\end{array}\right\} \quad \text { if }\left\{\lambda_{i}, \lambda_{i+1}\right\} \text { is a block, } \epsilon_{\lambda_{i}} \neq 0, \\
& \left.\begin{array}{l}
c_{i}:=\lambda_{i} / 2+2(i-1), \\
c_{i+1}:=\left(\lambda_{i}-4\right) / 2+2 i=c_{i}
\end{array}\right\} \quad \text { if }\left\{\lambda_{i}, \lambda_{i+1}\right\} \text { is a block, } \epsilon_{\lambda_{i}}=0 .
\end{aligned}
$$

The image of $(\lambda, \epsilon)$ under $i_{G}^{-1}$ is then the unique distinguished symbol with entries $c_{1}, \ldots, c_{r}$. Thus again $i_{G}$ restricts to a bijection $i_{G}: D_{n, 0}^{4} \stackrel{\sim}{\rightarrow} X_{G}$.

Finally, the map $\pi_{G}$ from the partially ordered set of unipotent classes $X_{G^{\prime}}$ of $\hat{G}^{\prime}$ in $G^{\prime}$ to the partially ordered set of unipotent classes of $\hat{G}$ contained in $G$ can be described as follows. Let $\lambda^{\prime}$ be the label of a unipotent class in $G^{\prime}$. Then $\pi_{G}\left(\lambda^{\prime}\right)$ has the label $(\lambda, \epsilon)$ where

$$
\lambda_{i}:= \begin{cases}\lambda_{i}^{\prime}-1 & \text { if } \lambda_{i}^{\prime} \text { is odd, } i \text { is even, } \lambda_{i-1}^{\prime}<\lambda_{i}^{\prime}, \\ \lambda_{i}^{\prime}+1 & \text { if } \lambda_{i}^{\prime} \text { and } i \text { are odd, } \lambda_{i}^{\prime}<\lambda_{i+1}^{\prime}, \\ \lambda_{i}^{\prime} & \text { otherwise, }\end{cases}
$$

and $\epsilon_{\lambda_{i}}=0$ if and only if $\lambda_{i}^{\prime}$ is even and $i$ is odd (see [19, III.7.2]). We have now given combinatorial descriptions for all maps in (2.3).

Proposition 2.19. Theorem 2.1 and Proposition 2.2 hold for groups of type $\mathrm{SO}_{2 n}$.

Proof. We first check the assertions for the sets of unipotent classes of $\hat{G}, \hat{G}^{\prime}$. We start with the statements in Theorem 2.1. By the description of $\psi_{G^{\prime}}$ and $\psi_{G}$ in (2.15) and (2.16), if $\psi_{G^{\prime}}(E)$ is distinguished for some $E \in \operatorname{Irr}\left(W_{n}\right)^{\prime}$, then so is $\psi_{G}(E)$. Denoting the entries of $\psi_{G^{\prime}}(E)$ by $\left(c_{1}^{\prime}, \ldots, c_{r}^{\prime}\right)$ we see from $(2.10)$ as in the previous proof that the parts of the partition $\lambda^{\prime}=i_{0}(E)$ are given by

$$
\begin{aligned}
& \lambda_{i}^{\prime}:=2 \alpha_{k}+1 \quad \text { if } i=2 k-1, \beta_{k-1}<\alpha_{k}<\beta_{k}-1, \\
& \lambda_{i}^{\prime}:=2 \beta_{k}-1 \quad \text { if } i=2 k, \alpha_{k}<\beta_{k}<\alpha_{k+1}+2 \text {, } \\
& \left.\begin{array}{l}
\lambda_{i}^{\prime}:=2 \beta_{k}-2, \\
\lambda_{i+1}^{\prime}:=2 \alpha_{k+1}+2=\lambda_{i}^{\prime}
\end{array}\right\} \quad \text { if } i=2 k, \beta_{k}=\alpha_{k+1}+2, \\
& \left.\begin{array}{l}
\lambda_{i}^{\prime}:=2 \alpha_{k}+1, \\
\lambda_{i+1}^{\prime}:=2 \beta_{k}-1=\lambda_{i}^{\prime}
\end{array}\right\} \quad \text { if } i=2 k-1, \beta_{k}=\alpha_{k}+1, \\
& \left.\begin{array}{l}
\lambda_{i}^{\prime}:=2 \alpha_{k}, \\
\lambda_{i+1}^{\prime}:=2 \beta_{k}=\lambda_{i}^{\prime}
\end{array}\right\} \quad \text { if } i=2 k-1, \beta_{k}=\alpha_{k} .
\end{aligned}
$$


For $(\lambda, \epsilon)=i_{G}(E)$ we obtain from $(2.17)$

$$
\begin{aligned}
& \lambda_{i}:=2 \alpha_{k}+2 \quad \text { if } i=2 k-1, \beta_{k-1}<\alpha_{k}<\beta_{k}-1, \\
& \lambda_{i}:=2 \beta_{k}-2 \\
& \lambda_{i}:=2 \beta_{k}-2, \\
& \left.\begin{array}{l}
\lambda_{i+1}:=2 \alpha_{k+1}+2=\lambda_{i} \\
\lambda_{i}:=2 \alpha_{k}+1, \\
\lambda_{i+1}:=2 \beta_{k}-1=\lambda_{i}
\end{array}\right\} \quad \text { if } i=2 k, \alpha_{k}<\beta_{k}<\alpha_{k+1}+2, \\
& \left.\begin{array}{l}
\lambda_{i}:=2 \alpha_{k}, \\
\lambda_{i+1}:=2 \beta_{k}=\lambda_{i}
\end{array}\right\} \quad \text { if } i=2 k, \beta_{k}=\alpha_{k+1}+2,
\end{aligned} \quad \text { if } i=2 k-1, \beta_{k}=\alpha_{k},
$$

and $\epsilon_{\lambda_{i}}=0$ only in the last case. The description of $\pi_{G}$ in (2.18) now allows to conclude that $\pi_{G}\left(i_{0}(E)\right)=i_{G}(E)$ in the cases considered. This proves part (1).

For part (2) the arguments are essentially the same as in the previous proof. Let $E \in \operatorname{Irr}\left(W_{n}\right)^{\prime}$ with label $(\alpha, \beta)$. We apply $R^{2,0}$ repeatedly to transform both $\Lambda^{\prime}:=\psi_{G^{\prime}}(E)$ and $\Lambda:=\psi_{G}(E)$ into distinguished symbols. Again, it is routine to check that the class corresponding to $R^{2,0}(\Lambda)$ is bigger than the class of $\Lambda$ in the partial ordering [19, I.2.10]. This proves (2).

To prove Proposition 2.2 for $G^{\prime}$ we can use $R^{1,0}$ and the description of families and special characters in [7, 4.6 and 4.7].

Finally, we descend to the unipotent classes of $G, G^{\prime}$. If a class of $\hat{G}^{\prime}$ splits into two $G^{\prime}$-classes, these have the same dimension, so the validity of $2.1(2)$ is not affected by this splitting. As for 2.1(1), it suffices to note that all splitting classes are induced in the sense of Lusztig and Spaltenstein. Thus by the property [20, 4.1] of the generalized Springer correspondence the Springer correspondent can be determined by Macdonald's $j$-induction in the Weyl group. In particular, it does not depend on the characteristic. On the other hand, the image of the map $\pi_{G}$ is determined by property [19, III.5.2(1)] by fusion of classes. But now by [19, II.7.6] the induced and the fused class are related in the same way, regardless of the characteristic. This shows that in fact property 2.1(1) also holds for the splitting unipotent classes of $G^{\prime}, G$.

By [7, 4.6 and 4.7] those characters in $\operatorname{Irr}\left(W_{n}^{\prime}\right)$ which do not extend to $W_{n}$ all lie in one-element families. Thus Proposition 2.2 is trivially satisfied for these characters. This completes the descent to the connected group $G^{\prime}$.

E. A combinatorial formula for $\operatorname{dim} \mathcal{B}_{u}$. Let $\Lambda \in X_{n, d}^{r, s}$ for $d \in\{0,1\}$ be a symbol. We define

$$
a(\Lambda):= \begin{cases}\sum_{\left\{c, c^{\prime}\right\}} \min \left(c, c^{\prime}\right)-r\left(\begin{array}{c}
m \\
2
\end{array}\right) \frac{4 m+1}{3}-s\left(\begin{array}{c}
m+1 \\
2
\end{array}\right) \frac{4 m-1}{3}, & \text { if } d=1, \\
\sum_{\left\{c, c^{\prime}\right\}} \min \left(c, c^{\prime}\right)-r\left(\begin{array}{c}
m \\
2
\end{array}\right) \frac{4 m-5}{3}-s\left(\begin{array}{c}
m \\
2
\end{array}\right) \frac{4 m+1}{3}, & \text { if } d=0,\end{cases}
$$

where the sum runs over all unordered pairs of entries of $\Lambda$ and $m \geq 0$ is the number of coefficients in the second component of $\Lambda$. First note that $a(\Lambda)$ is indeed well-defined on symbols. Furthermore, it is clear that $a$ is constant on similarity classes of symbols.

By the results of Lusztig and Spaltenstein quoted above, the sets of unipotent conjugacy classes of classical groups can be naturally parametrized by distinguished symbols in $X_{n, 1}^{1,1}, X_{n, 1}^{2,2}, Y_{n, 0}^{2}, Y_{n, 0}^{4}$, according to the cases. It thus makes sense to speak of the symbol $\Lambda_{u}$ associated to a unipotent class $C \ni u$ of a classical group. 
Proposition 2.23. Let $C$ be a unipotent conjugacy class of a classical group, $u \in C$ and $\Lambda_{u}$ be the associated distinguished symbol. Then

$$
\operatorname{dim} \mathcal{B}_{u}=a\left(\Lambda_{u}\right)
$$

Proof. This is a combinatorial exercise, using Spaltenstein's formulae for $\operatorname{dim} \mathcal{B}_{u}$. We give some details for the case of $\mathrm{Sp}_{2 n}$ in odd characteristic, where the corresponding distinguished symbols lie in $X_{n, 1}^{1,1}$. First, the class of regular unipotent elements has $\operatorname{dim} \mathcal{B}_{u}=0$ and the associated symbol is $((n),-)$, so the formula is correct in this case. Furthermore, $((n),-)$ is equivalent to the symbol $\Lambda:=((0,2,4, \ldots, 2 n, 3 n),(1,3,5, \ldots, 2 n+1))$, and by our above remark the $a$ value is constant on equivalence classes. Now every distinguished symbol in $X_{n, 1}^{1,1}$ can be obtained from $\Lambda$ by a sequence of steps in each of which only two adjacent entries are changed by plus and minus 1 . On the other hand, for $\lambda$ parametrizing a unipotent class of $\mathrm{Sp}_{2 n}$, the value of $\operatorname{dim} \mathcal{B}_{u}$ is given by

$$
\operatorname{dim} \mathcal{B}_{u}=\frac{1}{4} \sum_{i \geq 1}\left({\lambda_{2 i-1}^{*}}^{2}+\lambda_{2 i}^{*}\left(\lambda_{2 i}^{*}-2\right)\right),
$$

where $\lambda^{*}$ denotes the partition dual to $\lambda$ [19, II.6.3]. It remains to verify that the elementary steps described above give the same change in both formulae, using the connection (2.6) between $\lambda$ and the associated symbol. We omit further details.

Note that the sets $X_{n, 1}^{1,0}$ and $Y_{n, 0}^{1}$ have been used by Lusztig to parametrize unipotent characters of groups of classical type. In that context the value $a(\Lambda)$ gives the precise order of vanishing of the character degree, considered as a polynomial in $q$, at $q=0$.

\section{A formula For the AVERAge VAlue}

We assume from now on that $p>0$ and that $G$ is defined over the finite subfield of $k$ with $q$ elements, with corresponding Frobenius map $F: G \rightarrow G$. We also assume that the chosen maximal torus $T$ and the chosen Borel subgroup $B \supseteq T$ are $F$-stable.

Let $\rho \in \operatorname{Irr}\left(G^{F}\right)$ and $\rho_{\text {unif }}$ be the uniform projection of $\rho$. By [2, 1.3], we have $\operatorname{AV}(C, \rho)=\operatorname{AV}\left(C, \rho_{\text {unif }}\right)$ for all $F$-stable unipotent classes $C$ in $G$. The values of $\rho_{\text {unif }}$ at unipotent elements can be expressed in terms of Green functions, as follows.

For any $w \in W$ let $T_{w} \subseteq G$ be an $F$-stable maximal torus obtained by twisting $T$ with $w$ and let $Q_{w}$ be the Green function corresponding to $T_{w}$. For $E \in \operatorname{Irr}(W)^{F}$ let $Q_{E}=|W|^{-1} \sum_{w \in W} \operatorname{Trace}(F w, E) Q_{w}$ (cf. [16, (5.5)]), defined with respect to the 'preferred extension' [9, (17.2)] of $E$ to a module for the semi-direct product of $W$ with the cyclic subgroup of Aut $(W)$ generated by $F$. Since the Green functions are linearly independent, there exist unique constants $b(\rho, E) \in \mathbb{C}$ such that

$$
\rho_{\text {unif }}(g)=\sum_{E \in \operatorname{Irr}(W)^{F}} b(\rho, E) Q_{E}(g) \quad \text { for all unipotent } g \in G^{F} .
$$

Our strategy will be to get a hold on the coefficients $b(\rho, E)$ and the average values of the corresponding Green function $Q_{E}$. From now until (3.6) we will assume that the center of $G$ is connected. In the main result, Theorem 3.7, this condition will be removed using regular embeddings. 
A. Green functions and Springer correspondence. The Frobenius map $F$ acts on the set $\mathcal{N}_{G}$. With each $i=(C, \psi) \in \mathcal{N}_{G}^{F}$ we can associate a class function $Y_{i}$ on $G^{F}$ defined as follows. Since $i$ is $F$-stable, we have $F(C)=C$; furthermore, if $V$ is an $A_{G}(u)$-module affording the character $\psi$, there exists an automorphism $\alpha_{V}: V \rightarrow V$ of finite order such that $\alpha_{V} \circ y=F(y) \circ \alpha_{V}$ for all $y \in A_{G}(u)$. Then $Y_{i}(g)=0$ for $g \in G^{F} \backslash C^{F}$ and $Y_{i}\left(u_{y}\right)=\operatorname{Trace}\left(\alpha_{V} \circ y, V\right)$ for $y \in A_{G}(u)$. Note that this determines $Y_{i}$ only up to multiplication by a root of unity. The functions $Y_{i}$ (for the various $i \in \mathcal{N}_{G}^{F}$ ) form a basis of the space of class functions on $G^{F}$ supported on unipotent elements (cf. [10, (24.2.7)]).

The Springer correspondence for $G$ is compatible with the action of $F$ on $\operatorname{Irr}(W)$ and on $\mathcal{N}_{G}$. Hence it restricts to an injective map $i_{G}: \operatorname{Irr}(W)^{F} \rightarrow \mathcal{N}_{G}^{F}$. Let $E \in$ $\operatorname{Irr}(W)^{F}$ and $i_{G}(E)=(C, \psi) \in \mathcal{N}_{G}^{F}$. For $u \in C$ we denote by $\mathcal{B}_{u}$ the variety of Borel subgroups containing $u$, and let $d:=\operatorname{dim} \mathcal{B}_{u}$. Then, for a suitable normalization of the $Y_{i}$, we have that

$$
Q_{E}=q^{d} Y_{i_{G}(E)}+\text { linear combination of } Y_{\left(C^{\prime}, \psi^{\prime}\right)} \text { where } \operatorname{dim} C^{\prime}<\operatorname{dim} C .
$$

For large enough $p$, this is contained in [16]. In general, it follows by combining the methods in [10, Section 24], with [17, Theorem 5.5] and [2, Prop. 3.5]. Since the values of all Green functions are rational integers, we deduce from equation (3.1) that $Y_{i_{G}(E)}$ is also rational valued. This implies that, for any $F$-stable unipotent class $C$, there exists a sign $\varepsilon_{C}= \pm 1$ such that

$$
Y_{(C, 1)}(g)=\varepsilon_{C} \quad \text { for all } g \in C^{F} .
$$

Indeed, the pair $i_{0}:=(C, 1) \in \mathcal{N}_{G}$ always lies in the image of the Springer map $\operatorname{Irr}(W) \rightarrow \mathcal{N}_{G}$. By definition, the corresponding function $Y_{i_{0}}$ is a scalar multiple of the characteristic function on $C^{F}$. The fact that the values of this function are rational integers therefore implies (3.2).

In the following three results (3.3)-(3.5), the condition ' $p$ and $q$ are large enough' means that the results in [12] are applicable; in particular, the unipotent support of an irreducible character exists in this case.

Proposition 3.3. Assume that $p$ and $q$ are large enough. Let $\rho \in \operatorname{Irr}\left(G^{F}\right)$ and $C_{\rho}$ be its unipotent support. Let $E \in \operatorname{Irr}(W)^{F}$ and $i_{G}(E)=(C, \psi) \in \mathcal{N}_{G}^{F}$. If $b(\rho, E) \neq 0$, then $\operatorname{dim} C \leq \operatorname{dim} C_{\rho}$ with equality only if $C=C_{\rho}$.

Proof. Let $C$ be an $F$-stable unipotent class such that $i_{G}(E)=(C, \psi)$ for some $E, \psi$ and such that $b(\rho, E) \neq 0$. Assume that $C$ has maximal possible dimension with this property.

We claim that then $\rho_{\text {unif }}(g) \neq 0$ for some $g \in C^{F}$. To see this, consider any $E^{\prime} \in \operatorname{Irr}(W)^{F}$ such that $b\left(\rho, E^{\prime}\right) \neq 0$. Let $i_{G}\left(E^{\prime}\right)=\left(C^{\prime}, \psi^{\prime}\right)$. By the maximality condition on $C$ we have $\operatorname{dim} C^{\prime} \leq \operatorname{dim} C$. Using formula (3.1) we see that if $\operatorname{dim} C^{\prime}<$ $\operatorname{dim} C$, then $Q_{E^{\prime}}$ does not give any contribution to the restriction of $\rho_{\text {unif }}$ to $C^{F}$. On the other hand, if $C^{\prime}=C$, then the restriction of $Q_{E^{\prime}}$ to $C^{F}$ is a non-zero scalar times $Y_{i}$ where $i=i_{G}\left(E^{\prime}\right)$. We conclude that the restriction of $\rho_{\text {unif }}$ to $C^{F}$ is a non-zero linear combination of the functions $Y_{i}$, where $i=\left(C, \psi^{\prime}\right)$ for some $\psi^{\prime}$ and $i=i_{G}\left(E^{\prime}\right)$ for some $E^{\prime} \in \operatorname{Irr}(W)^{F}$ with $b\left(\rho, E^{\prime}\right) \neq 0$. Since the $Y$-functions are linearly independent, we conclude that that restriction can not be identically zero, and our claim is established.

Now the restriction of $\rho$ to $C^{F}$ is a linear combination of the restriction of $\rho_{\text {unif }}$ to $C^{F}$ and functions $Y_{i}$ where $i \in \mathcal{N}_{G}^{F}$ is not in the image of the map $i_{G}: \operatorname{Irr}(W)^{F} \rightarrow$ 
$\mathcal{N}_{G}^{F}$ (cf. $[2,(3.6)]$ ). Using once more that the $Y$-functions are linearly independent we conclude that the restriction of $\rho$ to $C^{F}$ can not be identically zero. Hence there exists some $g \in C^{F}$ such that $\rho(g) \neq 0$.

We can now use [12, Theorem 11.2] to conclude that $\operatorname{dim} C \leq \operatorname{dim} C_{\rho}$, with equality only for $C=C_{\rho}$. This completes the proof.

Corollary 3.4. Assume that $p$ and $q$ are large enough. Let $\rho \in \operatorname{Irr}\left(G^{F}\right)$ and $C_{\rho}$ be its unipotent support. Let $E_{0} \in \operatorname{Irr}(W)^{F}$ such that $i_{G}\left(E_{0}\right)=\left(C_{\rho}, 1\right) \in \mathcal{N}_{G}^{F}$. Then $\operatorname{AV}\left(C_{\rho}, \rho\right)= \pm q^{d}\left|A_{G}(u)\right| b\left(\rho, E_{0}\right)$, where $u \in C_{\rho}^{F}$ and $d=\operatorname{dim} \mathcal{B}_{u}$.

Proof. We have $\operatorname{AV}\left(C_{\rho}, \rho\right)=\operatorname{AV}\left(C_{\rho}, \rho_{\text {unif }}\right)$ (see [2, Prop. 1.3]). As above, let us write $\rho_{\text {unif }}(g)=\sum_{E} b(\rho, E) Q_{E}(g)$, for $g \in G^{F}$ unipotent. By Proposition 3.3 we see that

$$
\operatorname{AV}\left(C_{\rho}, \rho\right)=\sum_{E} b(\rho, E) \operatorname{AV}\left(C_{\rho}, Q_{E}\right)
$$

where the sum is over all $E \in \operatorname{Irr}(W)^{F}$ such that $i_{G}(E)=\left(C_{\rho}, \psi\right)$ for some $\psi$. The formula (3.1) shows that if $i_{G}(E)=\left(C_{\rho}, \psi\right)$, then the restriction of $Q_{E}$ to $C_{\rho}^{F}$ equals $q^{d} Y_{i_{G}(E)}$. In [2] the average value of any class function on a unipotent class $C$ is expressed in terms of the scalar product of that class function with a certain function $f_{C}^{\prime}$. Applying this to $C=C_{\rho}$ and the class function $Y_{i}$, and using the formulae in $[2,(2.1)]$ we see that the average value $\operatorname{AV}\left(C_{\rho}, Y_{i}\right)$ is zero unless $i=\left(C_{\rho}, 1\right)$ in which case it equals $\pm\left|A_{G}(u)\right|$ (cf. equation 3.2). Thus, we can conclude that $\operatorname{AV}\left(C_{\rho}, \rho\right)= \pm q^{d}\left|A_{G}(u)\right| b\left(\rho, E_{0}\right)$, as desired.

B. Series and families of irreducible characters. In order to get a hold on $b\left(\rho, E_{0}\right)$ we now have to recall some facts from [7, Chap. 4] concerning the classification of irreducible characters of $G^{F}$. Let $G^{*}$ be a group dual to $G$. Then $G^{*}$ is also defined over $\mathbb{F}_{q}$ and we denote again by $F$ the corresponding Frobenius map. Let $T^{*} \subseteq G^{*}$ be an $F$-stable maximal torus in duality with $T$. The Weyl group of $G^{*}$ with respect to $T^{*}$ can be canonically identified with $W$, where the actions of the Frobenius maps of $G$ and $G^{*}$ on $W$ are inverse to each other.

Let $s \in T^{*}$ be an element such that the $G^{*}$-conjugacy class of $s$ is $F$-stable, and let $W_{s} \subseteq W$ be the Weyl group of $C_{G^{*}}(s)$ with respect to $T^{*}$. The Frobenius map $F$ induces an automorphism $\gamma: W_{s} \rightarrow W_{s}$. Let $\operatorname{Irr}\left(W_{s}\right)^{\gamma}$ be the set of all $\gamma$-invariant irreducible characters of $W_{s}$. For each $E \in \operatorname{Irr}\left(W_{s}\right)^{\gamma}$ we have a corresponding almost character $R_{E}^{s}$, which is defined as a certain rational linear combination of Deligne-Lusztig generalized characters. (Note that almost characters are only welldefined up to sign, see [7, (3.7)]; a definitive choice of these signs is given in terms of the 'preferred extensions' in $[9,(17.2)]$.)

Let $\rho$ be an irreducible character of $G^{F}$. Then there exist some $s \in T^{*}$ and $E \in \operatorname{Irr}\left(W_{s}\right)^{\gamma}$ as above such that the scalar product between $\rho$ and $R_{E}^{s}$ is non-zero; if $s^{\prime} \in T^{*}$ is another such element, then $s, s^{\prime}$ are conjugate (see [7, (8.4.4)]). Thus, we can associate with $\rho$ a unique $F$-stable conjugacy class of semisimple elements in $G^{*}$. In this way, we have series of irreducible characters corresponding to the various $s \in T^{*}$ such that the class of $s$ in $G^{*}$ is $F$-stable.

Assume that $\rho \in \operatorname{Irr}\left(G^{F}\right)$ lies in the series defined by $s \in T^{*}$. If $E, E^{\prime} \in \operatorname{Irr}\left(W_{s}\right)^{\gamma}$ are such that $\rho$ has non-zero scalar product with $R_{E}^{s}$ and with $R_{E^{\prime}}^{s}$ then $E, E^{\prime}$ belong to the same family of characters of $W_{s}$ (see $[7,(5.25)$ and (6.17)]). Thus, we can 
associate with $\rho$ a unique $\gamma$-stable family of characters of $W_{s}$. Let $E_{1}$ be the unique special character in this family and $D_{E_{1}} \in \mathbb{Q}[X]$ its 'generic degree' (where $X$ is an indeterminate). As in [7, (4.1)], we define integers $f_{E_{1}} \neq 0$ and $a_{E_{1}} \geq 0$ by the condition that

$$
D_{E_{1}}(X)=f_{E_{1}}^{-1} X^{a_{E_{1}}}+\text { higher powers of } X .
$$

The number $a_{E_{1}}$ is canonically associated with $\rho$. In order to indicate this, we shall also denote it by $a_{\rho}$ and call it the generic q-part of $\rho(1)$. Finally, there exists a unique integer $n_{\rho} \geq 1$ such that the scalar product of $\rho$ and $R_{E_{1}}^{s}$ is given by $\pm n_{\rho}^{-1}$. We call $n_{\rho}$ the generic denominator in $\rho(1)$. These definitions are justified by the fact that we have

$$
\rho(1)= \pm n_{\rho}^{-1} q^{a_{\rho}} N
$$

where $N$ is an integer satisfying $N \equiv 1$ modulo $q$ (see [7, (4.26.3)]).

Proposition 3.5. Assume that $p$ and $q$ are large enough. Let $\rho \in \operatorname{Irr}\left(G^{F}\right)$ and $C_{\rho}$ be its unipotent support. Let $E_{0} \in \operatorname{Irr}(W)^{F}$ such that $i_{G}\left(E_{0}\right)=\left(C_{\rho}, 1\right) \in \mathcal{N}_{G}^{F}$. Then $b\left(\rho, E_{0}\right)= \pm n_{\rho}^{-1}$ and $a_{\rho}=\operatorname{dim} \mathcal{B}_{u}\left(\right.$ for $\left.u \in C_{\rho}\right)$.

Proof. We compute $\operatorname{AV}\left(C_{\rho}, \rho\right)$ in two different ways. On one hand, by Corollary 3.4, this equals $\pm q^{d}\left|A_{G}(u)\right| b\left(\rho, E_{0}\right)$, where $u \in C_{\rho}^{F}$ and $d=\operatorname{dim} \mathcal{B}_{u}$.

On the other hand, let $u_{1}, \ldots, u_{r} \in C_{\rho}^{F}$ be representatives for the $G^{F}$-classes contained in $C_{\rho}^{F}$ and $\Gamma_{0}=\sum_{j=1}^{r}\left[A_{G}\left(u_{j}\right): A_{G}\left(u_{j}\right)^{F}\right] \Gamma_{u_{j}}$ where $\Gamma_{u_{j}}$ denotes the generalized Gel'fand-Graev character associated with the unipotent element $u_{j} \in$ $C_{\rho}^{F}$. By $[12,(9.11)]$ and $\left[2\right.$, Theorem 1.4] we have $d=a_{\rho}$ and our average value is equal to $q^{d}$ times the scalar product between $\Gamma_{0}$ and $D_{G}(\bar{\rho})$ where $D_{G}$ denotes Alvis-Curtis-Kawanaka duality. Since these scalar products are integers, we can work with $\rho$ instead of $\bar{\rho}$. We conclude that it remains to show that the scalar product between $\Gamma_{0}$ and $D_{G}(\rho)$ equals $n_{\rho}^{-1}\left|A_{G}(u)\right|$, up to sign. In order to see this, we go through the proof of [12, Theorem 11.2].

Let $\left(s, E_{1}\right)$ be associated with $\rho$ as described above. Let $\mathcal{L}_{0}$ be an $F$-stable tame local system on $T$ in duality with $s \in T^{*}$, and let $\varepsilon$ be the sign character of $W_{s}$. We can associate with $\mathcal{L}_{0}$ and $E_{1} \otimes \varepsilon \in \operatorname{Irr}\left(W_{s}\right)^{F}$ an $F$-stable character sheaf $A=A_{E_{1} \otimes \varepsilon}^{\mathcal{L}_{0}}$ on $G$ and we can choose an isomorphism $\varphi: F^{*} A \rightarrow A$ so that the corresponding characteristic function $\chi_{A, \varphi}$ is a class function on $G^{F}$ of norm 1 . Then it is known that $\chi_{A, \varphi}$ coincides with $R_{E_{1} \otimes \varepsilon}^{s}$, up to a scalar multiple which is in fact a root of unity (see, for example, [4, Cor. 2.3.2] or [17]).

Equation (h) in the proof of $[12,(11.2)]$ now tells us that the scalar product of $\Gamma_{0}$ with $D_{G}(\rho)$ is the same as the scalar product of $\left|A_{G}(u)\right| \chi_{A, \varphi}$ with $D_{G}(\rho)$; hence it is the same as the scalar product of $\left|A_{G}(u)\right| R_{E_{1} \otimes \varepsilon}^{s}$ with $D_{G}(\rho)$, up to a root of unity. Since these scalar products must be integers, the root of unity is \pm 1 .

Finally, observe that $R_{E_{1} \otimes \varepsilon}^{s}= \pm D_{G}\left(R_{E_{1}}^{s}\right)$, see [7, (6.8.6)], and recall that $D_{G}$ is a self-adjoint involution. Hence the scalar product of $R_{E_{1} \otimes \varepsilon}^{s}$ with $D_{G}(\rho)$ is the same (up to sign) as the scalar product of $R_{E_{1}}^{s}$ with $\rho$, which is $\pm n_{\rho}^{-1}$. This completes the proof.

C. Lusztig's map from irreducible characters to unipotent classes. Recall the following construction from $[12,(10.5)]$ or $[7,(13.3)]$. Let $Y$ be the cocharacter group of the maximal torus $T$. Then $Y$ is naturally a module for $W$. Let $s \in T^{*}$ and $W_{s} \subseteq W$ be the stabilizer of $s$ in $W$. By restriction, $Y$ also is a module for 
$W_{s}$. Using the generalized Macdonald $j$-induction (see [13]), we can associate an irreducible character $E \in \operatorname{Irr}(W)$ with any special character $E_{1} \in \operatorname{Irr}\left(W_{s}\right)$. This is done as follows. Let $b_{E_{1}}$ be the smallest $r \geq 0$ for which $E_{1}$ appears with non-zero multiplicity in the $r$-th symmetric power of $Y$. Then the multiplicity of $E_{1}$ is in fact equal to 1. Moreover, there exists a unique $E \in \operatorname{Irr}(W)$ such that $E$ appears with non-zero multiplicity both in the character induced from $E_{1}$ and in the $b_{E_{1}}$-th symmetric power of the $W$-module $Y$. We shall write $E=j_{W_{s}}^{W}\left(E_{1}\right)$. Thus, we can define a map

$$
\Phi_{G}:\left\{\left(s, E_{1}\right) \mid s \in T^{*} \text { and } E_{1} \in \operatorname{Irr}\left(W_{s}\right) \text { special }\right\} \longrightarrow\{\text { unipotent classes in } G\}
$$

by associating with the pair $\left(s, E_{1}\right)$ the unipotent class $C$ satisfying $i_{G}(E)=(C, \psi)$ for some $\psi$. (This coincides with the map described in $[7,(13.3)]$ or $[12,(10.5)]$ in good characteristic.)

Let $\rho \in \operatorname{Irr}\left(G^{F}\right)$ and $\left(s, E_{1}\right)$ be associated with $\rho$ as described in (B) above. Let $E_{1}^{\prime} \in \operatorname{Irr}\left(W_{s}\right)^{\gamma}$ be the unique special character which lies in the same family as $E_{1} \otimes$ sign. It is easy to see that the class $C:=\Phi_{G}\left(s, E_{1}^{\prime}\right)$ is $F$-stable. Thus, we also obtain a map

$$
\xi_{G}: \operatorname{Irr}\left(G^{F}\right) \longrightarrow\{F \text {-stable unipotent classes in } G\},
$$

given by associating with $\rho$ the unipotent class $\Phi_{G}\left(s, E_{1}^{\prime}\right)$. (This coincides with the map described in $[7,(13.4)]$ or $[12,(11.1)]$ in good characteristic.) Using the results in the previous section we can now prove that the properties stated in [loc. cit.] remain valid in all characteristics.

Proposition 3.6. Let $s \in T^{*}$ and $E_{1} \in \operatorname{Irr}\left(W_{s}\right)$ be special. Furthermore, let $C$ be a unipotent class in $G$ and $d:=\operatorname{dim} \mathcal{B}_{u}($ for $u \in C)$.

(a) Let $E=j_{W_{s}}^{W}\left(E_{1}\right) \in \operatorname{Irr}(W)$. If $i_{G}(E)=(C, \psi) \in \mathcal{N}_{G}$, then $\psi=1$ and $d=b_{E_{1}}$.

(b) Let $\rho, \rho^{\prime} \in \operatorname{Irr}\left(G^{F}\right)$ such that $\rho^{\prime}= \pm D_{G}(\rho)$. If $C=\xi_{G}(\rho)$, then $d=a_{\rho^{\prime}}$.

Proof. If $p$ is large enough, the statement about $\psi$ in (a) holds by [12, Theorem 10.7(iii)]. Using the results in the previous section, this implies that it is also true for any $p$. More precisely, the argument goes as follows.

Let $\tilde{k}$ be an algebraically closed field, $\tilde{G}$ a connected reductive group over $\tilde{k}$, and $\tilde{T} \subseteq \tilde{G}$ a maximal torus so that the root data of $G$ and $\tilde{G}$ with respect to $T$ and $\tilde{T}$, respectively, are isomorphic. Then we can identify $W$ with the Weyl group of $\tilde{G}$. Assume that the characteristic of $\tilde{k}$ is large enough so that the results in [12] are applicable. We can then also find a semisimple element $\tilde{s}$ in the dual group so that the stabilizer $W_{\tilde{s}} \subseteq W$ can be identified with $W_{s}$ (see [1, Theorem 2.1]).

Consider also a group $G^{\prime}$ of the same type as $G$ but over an algebraically closed field of characteristic 0 , as in the previous section.

Now we can argue as follows. Let $i_{\tilde{G}}(E)=(\tilde{C}, \tilde{\psi}) \in \mathcal{N}_{\tilde{G}}$. By $[12$, Theorem 10.7(iii)] we have $\tilde{\psi}=1$. Since $\pi_{\tilde{G}}: X_{G^{\prime}} \rightarrow X_{\tilde{G}}$ is a bijection, there is a unique $C_{0} \in X_{G^{\prime}}$ such that $\pi_{\tilde{G}}\left(C_{0}\right)=\tilde{C}$. Let $E_{0} \in \operatorname{Irr}(W)$ such that $i_{0}\left(E_{0}\right)=\left(C_{0}, 1\right)$. Using Theorem 2.1(1), we conclude that $i_{\tilde{G}}\left(E_{0}\right)=\left(\pi_{\tilde{G}}\left(C_{0}\right), 1\right)=(\tilde{C}, 1)=i_{\tilde{G}}(E)$. Since the Springer correspondence is injective, we therefore have that $E_{0}=E$. Now we can apply once more Theorem 2.1 to pass from $G^{\prime}$ to $G$ and conclude that $i_{G}(E)=(C, 1)$, where $C=\pi_{G}\left(C_{0}\right)$.

Now consider the asserted equality $d=b_{E_{1}}$ in (a). Again, if $p$ is large enough, it holds by the results in $[6,(13.1 .1)$ and (13.3)]. By a similar argument as before, it 
then holds for all $p$, using the fact that Spaltenstein's map preserves the dimension of classes.

In order to prove (b), assume that $\left(s, E_{1}\right)$ corresponds to $\rho^{\prime}$ as in (B). Then $\left(s, E_{1}^{\prime}\right)$ corresponds to $\rho$ where $E_{1}^{\prime}$ is the special character in the same family as $E_{1} \otimes \operatorname{sign}$ (cf. the last part of the proof of Proposition 3.5). By definitions, we have $\Phi_{G}\left(s, E_{1}\right)=C=\xi_{G}(\rho)$. Since $a_{\rho^{\prime}}=a_{E_{1}}$ (see (B)) and $a_{E_{1}}=b_{E_{1}}$ (recall that $E_{1}$ is special), we are done using (a).

Before we state our main result, we extend the above constructions to the case where the center of $G$ is not necessarily connected. In that case, we can embed $G$ 'regularly' as a closed subgroup into a group $G_{0}$ over $\mathbb{F}_{q}$ with a connected center and such that $G, G_{0}$ have the same derived subgroup (cf. [11]). Clearly, the embedding $G \subseteq G_{0}$ defines a bijection between the $F$-stable unipotent classes of $G$ and $G_{0}$, respectively.

Let $\rho \in \operatorname{Irr}\left(G^{F}\right)$ and $\rho_{0} \in \operatorname{Irr}\left(G_{0}^{F}\right)$ such that $\rho$ appears with non-zero multiplicity in the restriction of $\rho_{0}$. By [11, Prop. 10] the restriction of every irreducible character of $G_{0}^{F}$ to $G^{F}$ is multiplicity-free. Let $m_{\rho} \geq 1$ be the total number of irreducible characters of $G^{F}$ which occur in the restriction of $\rho_{0}$. (Note that $m_{\rho}$ does not depend on the choice of $\rho_{0}$ and that $m_{\rho}$ is not divisible by $p$.) Then we let

$$
a_{\rho}:=a_{\rho_{0}}, \quad n_{\rho}:=m_{\rho} n_{\rho_{0}}, \quad \text { and } \quad C_{\rho}:=\Phi_{G_{0}}\left(s, E_{1}\right),
$$

where $\left(s, E_{1}\right)$ is associated with $\rho_{0}$ as in (B). Equivalently, we could define $C_{\rho}:=$ $\xi_{G_{0}}\left( \pm D_{G_{0}}\left(\rho_{0}\right)\right)$. Note that $C_{\rho}$ does not depend on the choice of $\rho_{0}$. It is readily checked that the formula in (B) for $\rho(1)$ remains valid.

Theorem 3.7. Let $G$ be any connected reductive group over $\mathbb{F}_{q}$, with corresponding Frobenius map F. Let $\rho \in \operatorname{Irr}\left(G^{F}\right)$ and $C_{\rho}$ be the $F$-stable unipotent class associated with $\rho$ as above. Then $C_{\rho}$ is the unipotent support of $\rho$ and we have

$$
\operatorname{AV}\left(C_{\rho}, \rho\right)= \pm n_{\rho}^{-1} q^{d}\left|A_{G}(u)\right|, \quad d=a_{\rho},
$$

where $u \in C_{\rho}^{F}$ and $d=\operatorname{dim} \mathcal{B}_{u}$.

In particular, this means that every irreducible character of a finite group of Lie type has a unipotent support (see $\S 5$ for the Suzuki and Ree groups).

Proof. Let $G \subseteq G_{0}$ be a regular embedding, and $\rho_{0} \in \operatorname{Irr}\left(G_{0}\right)^{F}$ such that $\rho$ occurs in the restriction of $\rho_{0}$ to $G^{F}$. Using the argument in [2, Lemma 5.1] we see that if $C$ is any $F$-stable unipotent class in $G$, then $\left|A_{G_{0}}(u)\right| \operatorname{AV}\left(C, \rho_{0}\right)=m_{\rho}\left|A_{G}(u)\right| \operatorname{AV}(C, \rho)$; note that $A_{G_{0}}(u)$ is a quotient of $A_{G}(u)$. In view of the above definitions of $C_{\rho}, a_{\rho}$, $n_{\rho}$, this implies that, in order to prove the theorem, we can assume that the center of $G$ is connected.

Recall from [2] that as far as average values on unipotent classes are concerned, we can work with $\rho_{\text {unif }}$ instead of $\rho$. As before let $b(\rho, E) \in \mathbb{Q}$ such that $\rho_{\text {unif }}(g)=$ $\sum_{E} b(\rho, E) Q_{E}(g)$ for all unipotent $g \in G^{F}$.

We associate with $\rho$ a semisimple element $s \in T^{*}$ and a special character $E_{1} \in$ $\operatorname{Irr}\left(W_{s}\right)^{\gamma}$ as described in (B) above. Let $\rho$ correspond to the element $\bar{x}_{\rho}$ in the parameter set $\bar{X}\left(W_{s}, \gamma\right)$ by a bijection as in [7, Theorem 4.23]. Then the constants $b(\rho, E)$ only depend on $W_{s}, \gamma, E$ and $\bar{x}_{\rho}$ (cf. [2, Cor. 3.8]). Hence these constants are 'independent of $q$ '. This means that we can choose a power $\tilde{q}$ of a large prime and a group $\tilde{G}$ defined over $\mathbb{F}_{\tilde{q}}$ of the same type as $G$, with the same Weyl group $W$ 
and the same action of the Frobenius map on $W$; furthermore, by [1, Theorem 3.3], we can choose $\tilde{q}$ in such a way that there exists a semisimple element $\tilde{s}$ in the dual group such that the stabilizer $W_{\tilde{s}} \subseteq W$ can be identified with $W_{s}$ and the induced action of the Frobenius map on it is the same as it was for $W_{s}$. Then we can also identify the corresponding parameter sets $\bar{X}\left(W_{\tilde{s}}, \tilde{\gamma}\right)$ and $\bar{X}\left(W_{s}, \gamma\right)$. Take an irreducible character $\tilde{\rho}$ of $\tilde{G}^{F}$ in the series defined by $\tilde{s}$ so that $\bar{x}_{\tilde{\rho}}$ corresponds to $\bar{x}_{\rho}$ under this identification. We then have constants $\tilde{b}(\tilde{\rho}, E)$ defined analogously as before. The fact that these are 'independent of $q$ ' means that we have

$$
\tilde{b}(\tilde{\rho}, E)=b(\rho, E) \text { for all } E \in \operatorname{Irr}(W)^{F} .
$$

We can certainly assume that $\tilde{q}$ was chosen large enough so that the results in [12] are applicable. Hence, in particular, the character $\tilde{\rho}$ has a unipotent support, which we denote by $\tilde{C}_{\tilde{\rho}}$. By [12, Theorem 11.2], $\tilde{C}_{\tilde{\rho}}$ is the class associated with $\tilde{\rho}$ in the same way as $C_{\rho}$ was associated with $\rho$ above.

Now let $G^{\prime}$ be a group over $\mathbb{C}$ as in Section 2, and let $\pi_{G}: X_{G^{\prime}} \rightarrow X_{G}$, $\pi_{\tilde{G}}: X_{G^{\prime}} \rightarrow X_{\tilde{G}}$ be Spaltenstein's maps between the respective partially ordered sets of unipotent classes. Since $\tilde{q}$ is a power of a large prime, $\pi_{\tilde{G}}$ is an isomorphism of partially ordered sets (cf. the remarks preceding Theorem 2.1). In order to simplify notation, we identify $X_{G^{\prime}}$ with $X_{\tilde{G}}$ and consider $\pi_{G}$ directly as a map $X_{\tilde{G}} \rightarrow X_{G}$. Then the argument in the proof of Proposition 3.6 shows that

$$
C_{\rho}=\pi_{G}\left(\tilde{C}_{\tilde{\rho}}\right) \in X_{G} .
$$

Since $\pi_{G}$ preserves dimensions, we have $\operatorname{dim} C_{\rho}=\operatorname{dim} \tilde{C}_{\tilde{\rho}}$.

Next we show that the assertion of Proposition 3.3 remains true. Let $C$ be any $F$-stable unipotent class in $G$ and $E \in \operatorname{Irr}(W)^{F}$ such that $b(\rho, E) \neq 0$ and $i_{G}(E)=(C, \psi)$ for some $\psi$. We claim that then

$$
\operatorname{dim} C \leq \operatorname{dim} C_{\rho} \quad \text { with equality only if } C=C_{\rho} .
$$

Indeed, let $i_{\tilde{G}}(E)=(\tilde{C}, \tilde{\psi}) \in \mathcal{N}_{\tilde{G}}^{F}$. Since $\tilde{b}(\tilde{\rho}, E)=b(\rho, E) \neq 0$, Proposition 3.3 implies that $\operatorname{dim} \tilde{C} \leq \operatorname{dim} \tilde{C}_{\tilde{\rho}}$ with equality only for $\tilde{C}=\tilde{C}_{\tilde{\rho}}$. By Theorem 2.1(2), we have $\operatorname{dim} C \leq \operatorname{dim} \tilde{C}$ with equality only for $C=\pi_{G}(\tilde{C})$. Combining this with the previous inequality we deduce that

$$
\operatorname{dim} C \leq \operatorname{dim} \tilde{C} \leq \operatorname{dim} \tilde{C}_{\tilde{\rho}}=\operatorname{dim} C_{\rho} .
$$

Moreover, if $\operatorname{dim} C=\operatorname{dim} C_{\rho}$, then we have equality everywhere. The equality $\operatorname{dim} C=\operatorname{dim} \tilde{C}$ implies that $C=\pi_{G}(\tilde{C})$ and the equality $\operatorname{dim} \tilde{C}=\operatorname{dim} \tilde{C}_{\tilde{\rho}}$ implies that $\tilde{C}=\tilde{C}_{\tilde{\rho}}$. Using (a), we conclude that $C=\pi_{G}(\tilde{C})=\pi_{G}\left(\tilde{C}_{\tilde{\rho}}\right)=C_{\rho}$. This completes the proof of (b).

Now we show that the assertion of Corollary 3.4 remains valid. Let $E_{0} \in \operatorname{Irr}(W)^{F}$ such that $i_{G}\left(E_{0}\right)=\left(C_{\rho}, 1\right) \in \mathcal{N}_{G}^{F}$. We claim that

$$
b\left(\rho, E_{0}\right)= \pm n_{\rho}^{-1} \neq 0 .
$$

To see this, let $\tilde{E}_{0} \in \operatorname{Irr}(W)^{F}$ such that $i_{\tilde{G}}\left(\tilde{E}_{0}\right)=\left(\tilde{C}_{\tilde{\rho}}, 1\right) \in \mathcal{N}_{\tilde{G}}^{F}$. Since $\pi_{G}\left(\tilde{C}_{\tilde{\rho}}\right)=C_{\rho}$ (cf. (a)), we have $i_{G}\left(\tilde{E}_{0}\right)=\left(C_{\rho}, 1\right)$ by Theorem 2.1(1), and since the Springer correspondence is injective, we conclude that $E_{0}=\tilde{E}_{0}$. Since $\tilde{b}\left(\tilde{\rho}, E_{0}\right)=b\left(\rho, E_{0}\right)$ and $n_{\rho}=n_{\tilde{\rho}}$, Corollary 3.4 implies (c).

Finally, it remains to check that $C_{\rho}$ is the unipotent support of $\rho$ and that $\operatorname{AV}\left(C_{\rho}, \rho\right)$ has the asserted form. 
Indeed, let $C^{\prime}$ be any $F$-stable unipotent class in $G$ such that $\operatorname{AV}\left(C^{\prime}, \rho\right) \neq$ 0 . Then there exists some $E \in \operatorname{Irr}(W)^{F}$ such that $b(\rho, E) \neq 0$ and such that $\operatorname{AV}\left(C^{\prime}, Q_{E}\right) \neq 0$. Let $i_{G}(E)=(C, \psi) \in \mathcal{N}_{G}^{F}$. Since $Q_{E}$ is non-zero on some element of $C^{\prime}$, we conclude that $\operatorname{dim} C^{\prime} \leq \operatorname{dim} C$ with equality only for $C=C^{\prime}$ (using once more the formula $(3.1))$. On the other hand, since $b(\rho, E) \neq 0$, we conclude using (b) that $\operatorname{dim} C \leq \operatorname{dim} C_{\rho}$ with equality only if $C=C_{\rho}$. Combining these two inequalities we see that $\operatorname{dim} C^{\prime} \leq \operatorname{dim} C_{\rho}$ with equality only for $C^{\prime}=C_{\rho}$.

We can now transfer the proof of Corollary 3.4, essentially word by word, to conclude that $\operatorname{AV}\left(C_{\rho}, \rho\right)= \pm q^{d}\left|A_{G}(u)\right| b\left(\rho, E_{0}\right)$ where $E_{0} \in \operatorname{Irr}(W)^{F}$ is such that $i_{G}\left(E_{0}\right)=\left(C_{\rho}, 1\right)$. Then (c) yields the desired form for our average value. This completes the proof.

Remark 3.8. What can we say about the sign in the above expression for $\operatorname{AV}\left(C_{\rho}, \rho\right)$ ?

Assume first that $p$ and $q$ are large enough so that the results in [12] are applicable. Then $\operatorname{AV}\left(C_{\rho}, \rho\right)=q^{d}\left(\Gamma_{0}, D_{G}(\rho)\right)$ where $\Gamma_{0}$ is a sum of certain generalized Gel'fand-Graev characters and $D_{G}$ denotes Alvis-Curtis-Kawanaka duality (cf. the proof of Proposition 3.5). This implies that $\operatorname{AV}\left(C_{\rho}, \rho\right)$ is $\varepsilon_{\rho}$ times a positive integer where $\varepsilon_{\rho}= \pm 1$ is such that $\varepsilon_{\rho} D_{G}(\rho)$ is an irreducible character. Comparing this with our formula in Theorem 3.7 we deduce that

$$
\operatorname{AV}\left(C_{\rho}, \rho\right)=\varepsilon_{\rho} n_{\rho}^{-1} q^{d}\left|A_{G}(u)\right|
$$

On the other hand, our average value is given in terms of the constant $b\left(\rho, E_{0}\right)$ and the average value of $Q_{E_{0}}$ on $C_{\rho}$, where $i_{G}\left(E_{0}\right)=\left(C_{\rho}, 1\right)$. In order to compute the latter value we need to determine the sign for the corresponding $Y$-function in (3.2). If $q$ is a power of a good prime, this problem is solved (by work of Springer, Shoji, and Beynon-Spaltenstein) and it yields that the sign is always 1; (see [16] and the references there). Since all the constants $b(\rho, E)$ are 'independent of $q$ ', we can conclude that the above formula remains valid whenever $q$ is a power of a good prime.

It seems likely that the above formula for $\operatorname{AV}\left(C_{\rho}, \rho\right)$ remains valid even if $q$ is a power of a bad prime, but at the moment we cannot prove this since the sign in the formula (3.2) does not yet seem to be known in general.

Remark 3.9. Let $G^{\prime}$ be a group of the same type as $G$ but defined over an algebraically closed field of characteristic 0 . Let $\pi_{G}: X_{G^{\prime}} \rightarrow X_{G}$ be Spaltenstein's map between the respective partially ordered sets of unipotent classes. Theorem 3.7 implies that the image of the map $\rho \mapsto C_{\rho}$ is contained in the image of $\pi_{G}$. Hence, in bad characteristic, there can exist $F$-stable unipotent classes in $G$ which are not the unipotent support of any irreducible character of $G^{F}$ (see $\mathrm{Sp}_{4}$ over a field of characteristic 2, for example). This is in contrast to the situation in good characteristic, where every $F$-stable unipotent class is the unipotent support of some irreducible character, see [7, (13.4.2)].

\section{Some Applications}

Throughout this section, let $G$ be any connected reductive group defined over $\mathbb{F}_{q}$, with corresponding Frobenius map $F$. We will now discuss some consequences of the existence of unipotent supports, and give examples. 
A. Induction of classes. Let $L \subseteq G$ be a closed subgroup which is the Levi complement in some parabolic subgroup of $G$. Following Lusztig-Spaltenstein [13] we have an operation of induction of unipotent classes from $L$ to $G$, defined as follows. Choose a parabolic subgroup $P \subseteq G$ such that $P=U_{P} L$ where $U_{P}$ is the unipotent radical of $P$. Given any unipotent class $C$ in $L$, there exists a unique unipotent class $\hat{C}$ in $G$ such that $\hat{C} \cap C U_{P}$ is dense in $C U_{P}$. By [13, Theorem 2.2] the class $\hat{C}$ does not depend on the chosen parabolic subgroup $P$ containing $L$. Hence we can write $\hat{C}=\operatorname{Ind}_{L}^{G}(C)$.

Now assume that $L$ is $F$-stable. If, as above, $\hat{C}$ is induced from $C$ using the parabolic subgroup $P$, then $F(\hat{C})$ is induced from $F(C)$ using the parabolic subgroup $F(P)$. So, if $C$ is $F$-stable, then $\hat{C}$ must be $F$-stable, too. Thus, the induction takes $F$-stable classes in $L$ to $F$-stable classes in $G$.

Denote by $R_{L}^{G}$ the operation of twisted induction, see [5]; it maps virtual characters of $L^{F}$ to virtual characters of $G^{F}$. This operation preserves series of characters, in the following sense. The dual group $L^{*}$ can be identified with a closed subgroup in $G^{*}$. Let $s \in L^{*}$ be semisimple and $F$-stable. Then $s$ defines a series of characters of $L^{F}$ and of $G^{F}$, respectively. If $\rho \in \operatorname{Irr}\left(L^{F}\right)$ lies in the series defined by $s$, then $R_{L}^{G}(\rho)$ is an integral linear combination of irreducible characters in the series of $G^{F}$ defined by $s$ (see [5, Cor. 6]).

Assume furthermore that $C_{G^{*}}(s) \subseteq L^{*}$. Then there exists a sign $\varepsilon$ such that $\varepsilon R_{L}^{G}$ defines a bijection between the irreducible characters in the series of $L^{F}$ and $G^{F}$ defined by $s$, respectively (see [5, Prop. 10]). The following result shows that, in this situation, the notion of unipotent support is compatible with twisted induction of characters and induction of unipotent classes.

Proposition 4.1. In the above set-up, assume that $F(s)=s$ and that $C_{G^{*}}(s) \subseteq$ $L^{*}$. Let $\rho \in \operatorname{Irr}\left(L^{F}\right)$ be in the series defined by $s$ and let $\hat{\rho}:= \pm R_{L}^{G}(\rho) \in \operatorname{Irr}\left(G^{F}\right)$. If $C$ is the unipotent support of $\rho$, then $\operatorname{Ind}_{L}^{G}(C)$ is the unipotent support of $\hat{\rho}$.

Proof. Since induction of classes and twisted induction of characters are compatible with regular embeddings (cf. [11]), we may assume that the center of $G$ is connected. It will be convenient to use a slightly different set-up for the description of the $R_{L}^{G}$ map. This can be done as follows, see [7, (6.21)]. Let $P \subseteq G$ be a parabolic subgroup with Levi decomposition $P=U_{P} L$. There exist some $g \in G$ and a subset $J \subseteq S$ such that $g P g^{-1}=P_{J}$ and $g L g^{-1}=L_{J}$, where $P_{J}$ and $L_{J}$ denote the standard parabolic subgroup and the standard Levi complement defined by $J$, respectively. Then $g^{-1} F(g) \in N_{G}(T)$; denote this element by $\dot{w}_{2}$ and let $w_{2} \in W$ be its image under the canonical map $N_{G}(T) \rightarrow W$. Then $F\left(w_{2} J w_{2}^{-1}\right)=J$ and the map $F^{\prime}: l \mapsto F\left(\dot{w}_{2} l \dot{w}_{2}^{-1}\right)$ can be considered as a Frobenius map on $L_{J}$ such that the finite groups $L^{F}$ and $L_{J}^{F^{\prime}}$ are isomorphic. We can therefore regard $R_{L}^{G}$ as a map from virtual characters of $L_{J}^{F^{\prime}}$ to virtual characters of $G^{F}$. This description has the advantage that now we can assume that $s \in T^{*}$ and the $L_{J}^{*}$-conjugacy class of $s$ is $F^{\prime}$-stable. Let $W_{J} \subseteq W$ be the parabolic subgroup corresponding to $J \subseteq S$. Under the identification of $W$ with the Weyl group of $G^{*}$, the group $W_{J}$ is the Weyl group of $L_{J}^{*}$. Our assumption that $C_{G^{*}}(s) \subseteq L^{*}$ now translates to the condition that

$$
W_{s} \subseteq W_{J} \subseteq W .
$$

Let $\gamma^{\prime}: W_{s} \rightarrow W_{s}$ be the automorphism induced by $F^{\prime}$ in the same way as $\gamma: W_{s} \rightarrow W_{s}$ was induced by $F$. As remarked in [7, p.214] we have $\gamma=\gamma^{\prime}$. By 
Section 3(B), we can associate with $\rho$ a pair $\left(s, E_{1}\right)$ where $E_{1} \in \operatorname{Irr}\left(W_{s}\right)^{\gamma}$ is a special character. We claim that $\left(s, E_{1}\right)$ is also the pair associated with $\hat{\rho}= \pm R_{L}^{G}(\rho)$.

Indeed, for any $E \in \operatorname{Irr}\left(W_{s}\right)^{\gamma}$, let $R_{E}^{s, J}$ and $R_{E}^{s}$ be the corresponding almost characters of $L_{J}^{F^{\prime}}$ and $G^{F}$, respectively. Using the formulae in [7, (6.21.3) and (6.21.4)] we see that

$$
R_{L}^{G}\left(R_{E}^{s, J}\right)=R_{E}^{s}
$$

Moreover, $\rho$ appears with non-zero multiplicity in $R_{E}^{s, J}$ if and only if $\hat{\rho}$ appears with non-zero multiplicity in $R_{E}^{s}$. (The multiplicities are even the same up to a sign, by [5, Prop. 10].) Applying this to the special character $E_{1}$ and recalling the description in Section 3(B), the claim is proved.

Now we can proceed as follows. We regard $\rho$ as a character of $L_{J}^{F^{\prime}}$ and let $C$ be its unipotent support in $L_{J}$. By Theorem 3.7, we have

$$
i_{L_{J}}(E)=(C, 1) \quad \text { where } \quad E:=j_{W_{s}}^{W_{J}}\left(E_{1}\right) \in \operatorname{Irr}\left(W_{J}\right) .
$$

On the other hand, let $\hat{C}$ be the unipotent support of $\hat{\rho}$. Then we also have

$$
i_{G}(\hat{E})=(\hat{C}, 1) \quad \text { where } \hat{E}:=j_{W_{s}}^{W}\left(E_{1}\right) \in \operatorname{Irr}(W) .
$$

Since the $j$-operation is transitive, we conclude that $j_{W_{J}}^{W}(E)=\hat{E}$. Now we are in the set-up of $[20,4.1]$. So we can deduce that $\hat{C}=\operatorname{Ind}_{L}^{G}(C)$, and the proof is complete.

B. Unipotent characters and the Jordan decomposition. Recall from [7] that $\rho \in \operatorname{Irr}\left(G^{F}\right)$ is called unipotent if $\rho$ lies in the series defined by $s=1 \in T^{*}$. In this case, the description of Lusztig's map in Section 3(C) becomes slightly simpler. Indeed, let $E_{1}$ be the unique $F$-stable special character of $W$ such that the scalar product of $\rho$ with the almost character $R_{E_{1}}^{1}$ is non-zero. By Theorem 3.7 and Proposition 3.6 we have $i_{G}\left(E_{1}\right)=(C, 1)$ and $C$ is the unipotent support of $\rho$.

Let $X_{G}^{0}$ be the set of all unipotent classes $C$ in $G$ such that $i_{G}(E)=(C, 1)$ for some special character $E \in \operatorname{Irr}(W)$. By Theorem 2.1, this set is 'independent of the characteristic'; that is, if we also define it with respect to a group $G^{\prime}$ over $\mathbb{C}$ and of the same type as $G$, then Spaltenstein's map $\pi_{G}$ induces a bijection between $X_{G^{\prime}}^{0}$ and $X_{G}^{0}$. The classes in $X_{G}^{0}$ are called special. We have just seen that the unipotent support of a unipotent character is special.

The following characterization of families of unipotent characters was already conjectured by Lusztig [6].

Proposition 4.2. Two unipotent characters of $G^{F}$ belong to the same family if and only if they have the same unipotent support.

Proof. Let $\rho, \rho^{\prime}$ be unipotent characters, $E_{1}, E_{1}^{\prime}$ the special characters $\operatorname{in} \operatorname{Irr}(W)$ associated with $\rho, \rho^{\prime}$, and $C, C^{\prime}$ be the unipotent supports of $\rho, \rho^{\prime}$, respectively. The characters $\rho, \rho^{\prime}$ belong to the same family if and only if $E_{1}=E_{1}^{\prime}$. On the other hand, as remarked above, we have $i_{G}\left(E_{1}\right)=(C, 1)$ and $i_{G}\left(E_{1}^{\prime}\right)=\left(C^{\prime}, 1\right)$. Hence $E_{1}=E_{1}^{\prime}$ if and only if $C=C^{\prime}$.

Proposition 4.3. Let $\rho$ be a unipotent character of $G^{F}$ and $C_{\rho}$ be its unipotent support. If $C$ is any $F$-stable unipotent class in $G$ such that $\operatorname{AV}(C, \rho) \neq 0$, then $C \leq C_{\rho}$. (Recall that this means that $C$ is contained in the Zariski closure of $C_{\rho}$.) 
Proof. As above let $E_{1} \in \operatorname{Irr}(W)^{F}$ be the unique special character such that the scalar product between $\rho$ and $R_{E_{1}}^{1}$ is non-zero; then $i_{G}\left(E_{1}\right)=\left(C_{\rho}, 1\right)$. Since we are interested in average values on unipotent classes, we can replace $\rho$ by $\rho_{\text {unif }}$. By [7, 4.23], $\rho_{\text {unif }}$ is a linear combination of almost characters $R_{E}^{1}$ where $E \in \operatorname{Irr}\left(W^{F}\right)$ is in the same family as $E_{1}$. Using the formula (3.1) we see that it is sufficient to prove the assertion only for those $C$ such that $i_{G}(E)=(C, \psi)$ and $E$ is in the same family as $E_{1}$. Hence we are done by Proposition 2.2 .

Remark. It is intriguing to speculate that the assertion of Proposition 4.3 holds for all irreducible characters, and not only for the unipotent ones. In the same way as the above proposition follows from a property of the Springer correspondence (namely, Proposition 2.2), such a more general result would follow from a more general version of that property of the Springer correspondence: namely, it would have to be possible to formulate the assertions in [12, Corollary 10.9] in terms of the usual order relation on unipotent classes, and not in terms of dimensions. We hope to settle this question (in the positive or in the negative) elsewhere.

Assume that the center of $G$ is connected. Recall from [7, p.x] that we then have a Jordan decomposition of characters: let $s \in G^{*}$ be semisimple and $F$-stable, and $G(s)$ be a group over $\mathbb{F}_{q}$ 'dual' to $C_{G^{*}}(s)$ (see $[7,(8.4)]$ ). Then there exists a bijection between the irreducible characters of $G^{F}$ in the series defined by $s$ and the unipotent characters of $G(s)^{F}$; moreover, the multiplicities of corresponding characters in the almost characters $R_{E}^{s}, R_{E}^{1}$ defined with respect to $G$ and $G(s)$, respectively, are the same (up to a sign depending only on $s$ ). If $C_{G^{*}}(s)$ is the Levi complement of some parabolic subgroup of $G^{*}$, then the Jordan decomposition is given by the $R_{L}^{G}$-map and Proposition 4.1 shows that the unipotent supports of corresponding characters are related by the induction of classes as defined in [13]. In the general case, we have to work with Spaltenstein's generalized induction [19, III.12].

Lemma 4.4. Let $d_{0}: X_{G} \rightarrow X_{G}$ be the unique duality operation (in the sense of $\left[19\right.$, III.1.2]) for which the image $d_{0}\left(X_{G}\right)$ has minimal possible cardinality (cf. [19, III.9.4]). Then $X_{G}^{0}=d_{0}\left(X_{G}\right)$.

Proof. By standard reduction arguments we may assume that $G$ is a simple group over $\mathbb{C}$. If $G$ is of exceptional type, the assertion follows from the tables giving the Springer correspondence [20] and the description of $d_{0}$ in [19, III.9.1].

If $G$ is of type $A_{n}$, all characters of the Weyl group and all unipotent classes are special and $d_{0}$ is surjective, so the assertion is obvious.

Now let $G=\mathrm{Sp}_{2 n}$ be of type $C_{n}$, with Weyl group $W_{n}$. An irreducible character $E \in \operatorname{Irr}\left(W_{n}\right)$ parametrized by the pair of partitions $(\alpha, \beta)$ (normalized so that $\alpha$ has one part more than $\beta)$ is special if and only if $\Lambda^{\prime}=(\alpha, \beta)+\Lambda_{0,1}^{1,0} \in X_{n, 1}^{1,0}$ is distinguished $[7,4.5]$. By 2(B) the Springer correspondent of $E$ is the unipotent class with symbol $\Lambda=\Lambda^{\prime}+\Lambda_{0,1}^{0,1}=(\alpha, \beta)+\Lambda_{0,1}^{1,1} \in X_{n, 1}^{1,1}$. Let $\left(c_{1}, \ldots, c_{2 m+1}\right)$ denote the entries of $\Lambda$. Since $\Lambda^{\prime}$ is distinguished, it follows that $c_{i}=c_{i+1}$ implies that $i$ is even. By (2.6) this means that between any two consecutive odd entries of $\lambda:=i_{0}(E)$ and also above the largest odd entry of $\lambda$ there is an even number of even entries. This in turn shows that the dual partition $\lambda^{*}$ also parametrizes a unipotent class of $G$, and hence $\lambda \in d_{0}\left(X_{G}\right)$ by [19, III.3.11]. On the other hand, if $\lambda \in d_{0}\left(X_{G}\right)$, then it is easy to see that the distinguished symbol $\Lambda \in X_{n, 1}^{1,1}$ with 
$\lambda=i_{0}(\Lambda)$ is such that $\Lambda-\Lambda_{0,1}^{0,1}$ is still distinguished, so $\lambda$ is the image under the Springer map of a special character.

Next, let $G=\mathrm{SO}_{2 n+1}$ be of type $B_{n}$. Again, an irreducible character $E=$ $(\alpha, \beta) \in \operatorname{Irr}\left(W_{n}\right)$ is special if and only if $\Lambda^{\prime}=(\alpha, \beta)+\Lambda_{0,1}^{1,0} \in Y_{n, 1}^{1}$ is distinguished $[7,4.5]$. Let $\Lambda=(\alpha, \beta)+\Lambda_{0,1}^{2,0} \in X_{n, 1}^{2}$ with entries $\left(c_{1}, \ldots, c_{2 m+1}\right)$ denote the symbol of the Springer correspondent of $E$. Since $\Lambda^{\prime}$ is distinguished, it follows that $c_{i}=c_{i+1}$ implies that $i$ is odd. By (2.10) this means that between any two consecutive even entries of $\lambda:=i_{0}(E)$ there is an even number of odd entries, and above the largest even entry there is an odd number of odd entries. Thus the dual partition $\lambda^{*}$ also parametrizes a unipotent class of $G$, and hence $\lambda \in d_{0}\left(X_{G}\right)$ by [19, III.3.11]. The converse is also clear.

Finally, assume that $G=\mathrm{SO}_{2 n}$ is of type $D_{n}$ with Weyl group $W_{n}^{\prime}$. An irreducible character $E=(\alpha, \beta) \in \operatorname{Irr}\left(W_{n}^{\prime}\right)$ (normalized so that $\alpha$ and $\beta$ have the same number of parts) is special if and only if $\Lambda^{\prime}=(\alpha, \beta)+\Lambda_{0,0}^{1,0} \in Y_{n, 0}^{1}$ is distinguished [7, 4.6 and 4.7]. According to 2(D) let $\Lambda=(\alpha, \beta)+\Lambda_{0,0}^{2,0} \in Y_{n, 0}^{2}$ with entries $\left(c_{1}, \ldots, c_{2 m}\right)$ denote the symbol of the Springer correspondent of $E$. Since $\Lambda^{\prime}$ is distinguished, it follows that $c_{i}=c_{i+1}$ implies that $i$ is odd. By (2.10) this means that between any two consecutive even entries of $\lambda:=i_{0}(E)$ and also above the largest even entry of $\lambda$ there is an even number of odd entries. It follows from [19, III.3.10] that $e(\lambda)=\lambda$, and hence $\lambda \in d_{0}\left(X_{G}\right)$ since $e=d_{0}^{2}$. The converse is again obvious. This completes the proof.

Proposition 4.5. In the above set-up, let $\rho$ be an irreducible character of $G^{F}$ in the series defined by $s$, and $\rho_{1}$ be the corresponding unipotent character of $G(s)^{F}$. Let $C_{1}$ be the unipotent support of $\rho_{1}$. Then the unipotent support of $\rho$ is given by $j_{G(s), G}\left(C_{1}\right)$ where $j_{G(s), G}$ denotes Spaltenstein's generalized induction [19, III.12].

Proof. First note that the Weyl group $W_{s}$ of $C_{G^{*}}(s)$ (together with the action of $F$ on it) can be identified with the Weyl group of $G(s)$. By the remarks preceding Proposition 4.2, we have $i_{G(s)}\left(E_{1}\right)=\left(C_{1}, 1\right)$ where $E_{1} \in \operatorname{Irr}\left(W_{s}\right)^{F}$ is the unique special character such that $\rho_{1}$ has non-zero multiplicity in the almost character $R_{E_{1}}^{1}$ of $G(s)^{F}$. Furthermore, by the constructions in Section 3(C) and Theorem 3.7, the unipotent support of $\rho$ is the unique unipotent class $C$ such that $i_{G}\left(j_{W_{s}}^{W}\left(E_{1}\right)\right)=$ $(C, 1)$.

We can express this by the equality $C=j_{G(s), G}\left(C_{1}\right)$, where $j_{G(s), G}: X_{G(s)}^{0} \rightarrow X_{G}$ is the map obtained by composing the map $\Phi_{G}$ in Section $3(\mathrm{C})$ with the bijective correspondence (given by the Springer correspondence) between the special unipotent classes of $G(s)$ and the special characters in $\operatorname{Irr}\left(W_{s}\right)$. Note that this map $j_{G(s), G}$ is 'independent of the characteristic' (cf. the remarks preceding Proposition 4.2).

It remains to show that the family of maps $\left\{j_{G(s), G} \mid s \in G^{*}\right.$ semisimple $\}$ satisfies the conditions in [19, III.12.3] for generalized induction maps. By standard reduction arguments we may assume that $G$ is a simple algebraic group; note also that the Frobenius map $F$ does not play a role for this. Moreover, we can assume that we are in good characteristic (or that $G$ is a group over $\mathbb{C}$ ). By Lemma 4.4, each map $j_{G(s), G}$ is defined on the 'correct' subset of unipotent classes of $G(s)$. We now have to check that the five characterizing conditions in [19, III.12.3] hold for $j_{G(s), G}$.

Condition (I) is clear by Lemma 4.4. Conditions (IV) and (V) hold by the remarks in $[7,(13.3)]$ concerning the map $\Phi_{G}$. Now consider condition (II) and, 
instead of condition (III), the condition (III') described in [19, III.12.6]: these are satisfied by the transitivity of the Macdonald $j$-induction and the fact (already used at the end of the proof of Proposition 4.1) that the $j$-induction from a Levi subgroup corresponds to the usual induction of unipotent classes. Thus, conditions (I), (II), $\left(\mathrm{III}^{\prime}\right),(\mathrm{IV})$ and $(\mathrm{V})$ are satisfied for the maps $j_{G(s), G}$. If $G$ is a classical group, we are done by [19, III.12.6]. If $G$ is an exceptional group, it remains to check that $j_{G(s), G}$ yields the same results as in the table in [19, p.221]. We have done this using induce/restrict matrices between the characters of the corresponding Weyl groups and the tables for the Springer correspondence in [20]. This completes the proof.

C. Cuspidal characters and cuspidal classes. The Frobenius map $F: G \rightarrow G$ can be decomposed in the form $F=F_{q} \circ F_{0}=F_{0} \circ F_{q}$ where $F_{q}$ acts as multiplication by $q$ on the character group of $T$, while $F_{0}$ acts as an automorphism of finite order leaving the root system invariant.

Now let $G^{\prime}$ be a group of the same type as $G$ but over an algebraically closed field of characteristic 0, as in Section 2. Let $T^{\prime} \subseteq B^{\prime} \subseteq G^{\prime}$ correspond to $T \subseteq B \subseteq G$. Then we also have an automorphism $F_{0}: G^{\prime} \rightarrow G^{\prime}$ such that $F_{0}$ and $F_{0}^{\prime}$ act in the same way on the character groups of $T$ and $T^{\prime}$, respectively.

Let $\pi_{G}: X_{G^{\prime}} \rightarrow X_{G}$ be Spaltenstein's map between the partially ordered sets of unipotent classes in $G^{\prime}$ and $G$, respectively. The maps $F_{0}^{\prime}$ and $F$ induce bijections $F_{0}^{\prime}: X_{G^{\prime}} \rightarrow X_{G^{\prime}}$ and $F: X_{G} \rightarrow X_{G}$. Then the following diagram is commutative:

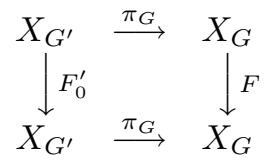

(Indeed, let $C \in X_{G}$ and $E \in \operatorname{Irr}(W)$ such that $i_{G}(E)=(C, 1) \in \mathcal{N}_{G}$. Since $F_{q}$ certainly acts trivially on $W$ and the Springer correspondence is compatible with the actions of $F$ on $\operatorname{Irr}(W)$ and on $\mathcal{N}_{G}$ we conclude that $F_{q}(C)=C$. This implies the commutativity of the above diagram.) In analogy to [3, Def. 3.1] we say that an $F_{0}^{\prime}$-stable unipotent class $C \in X_{G^{\prime}}$ is non-cuspidal (with respect to $F_{0}^{\prime}$ ) if there exist an $F_{0}^{\prime}$-stable proper parabolic subgroup $P^{\prime} \subset G^{\prime}$ and an $F_{0}^{\prime}$-stable Levi complement $L^{\prime} \subseteq P^{\prime}$ such that $C$ has non-trivial intersection with $L^{\prime}$ and such that, if we take an $F_{0}^{\prime}$-fixed element in this intersection, we have $A_{G^{\prime}}(u) \cong A_{L^{\prime}}(u)$. If no such parabolic subgroup exists, we say that $C$ is cuspidal.

Note that if $p$ is a good prime, $\pi_{G}$ is an isomorphism and we also have $A_{G^{\prime}}\left(u^{\prime}\right) \cong$ $A_{G}(u)$ where $u^{\prime} \in C$ for some $C \in X_{G^{\prime}}$ and $u \in \pi_{G}(C)$. It follows easily, using the above commutative diagram, that $\pi_{G}$ defines a bijection between the cuspidal unipotent classes of $G^{\prime}$ and the cuspidal unipotent classes of $G$ (in the sense of [3]). Thus, in good characteristic, the notions of cuspidal unipotent classes for $G^{\prime}$ and $G$ coincide.

Proposition 4.6. Let $\rho \in \operatorname{Irr}\left(G^{F}\right)$ be cuspidal and $C_{\rho}$ be its unipotent support. Then $C_{\rho}$ is the image under $\pi_{G}$ of a cuspidal class in $G^{\prime}$ (with respect to $F_{0}^{\prime}$ ).

Proof. Assume first that $p$ and $q$ are large enough so that the results in [12] are applicable. As in the proof of Proposition 3.5 (see also $[12,(9.11)]$ ) we can then express the average value of $\rho$ on $C_{\rho}$ in the form:

$$
\operatorname{AV}\left(C_{\rho}, \rho\right)=q^{d}\left(D_{G}\left(\Gamma_{0}\right), \rho\right),
$$


where $\Gamma_{0}$ is a sum of certain generalized Gel'fand-Graev characters. Assume, if possible, that $C_{\rho}$ is non-cuspidal. Then $C_{\rho}$ has non-trivial intersection with an $F$ stable Levi subgroup $L$ in some proper $F$-stable parabolic subgroup of $G$. Moreover, by [3, Corollary 2.7], we have

$$
\left(D_{G}\left(\Gamma_{0}\right), \rho\right)=\left(R_{L}^{G}\left(D_{L}\left(\Gamma_{0}^{L}\right)\right), \rho\right),
$$

where $R_{L}^{G}$ denotes Harish-Chandra induction and $\Gamma_{0}^{L}$ is a similar sum as above of generalized Gel'fand-Graev characters of $L^{F}$. Since $\operatorname{AV}\left(C_{\rho}, \rho\right) \neq 0$, we conclude that the scalar product on the right-hand side is non-zero. By definition, this means that $\rho$ is not cuspidal, a contradiction. This completes the proof in the case where $p$ and $q$ are large enough.

In the general case, let $\tilde{G}, \tilde{\rho}$ and $\tilde{C}_{\tilde{\rho}}$ be as in the proof of Theorem 3.7. Since $\rho$ and $\tilde{\rho}$ correspond to the same element in the parameter set $\bar{X}\left(W_{s}, \gamma\right)$, the characterization of cuspidal characters in [7, Lemma 6.3] shows that $\tilde{\rho}$ is a cuspidal character of $\tilde{G}^{F}$. The above discussion can be applied to $\tilde{G}$ and hence $\tilde{C}_{\tilde{\rho}}$ is cuspidal.

Let $\pi_{G}: X_{G^{\prime}} \rightarrow X_{G}$ and $\pi_{\tilde{G}}: X_{G^{\prime}} \rightarrow X_{\tilde{G}}$ be Spaltenstein's maps. As in the proof of Theorem 3.7, we have $\pi_{G}(C)=C_{\rho}$ and $\tilde{C}_{\tilde{\rho}}=\pi_{\tilde{G}}(C)$ for a unique class $C \in X_{G^{\prime}}$. We have seen that $\tilde{C}_{\tilde{\rho}}$, and hence also $C$, are cuspidal classes. So $C_{\rho}$ is the image of a cuspidal class in $G^{\prime}$ under $\pi_{G}$.

Remark. We have remarked above that, in good characteristic, Spaltenstein's map $\pi_{G}: X_{G^{\prime}} \rightarrow X_{G}$ defines a bijection between the cuspidal unipotent classes in $G^{\prime}$ and those in $G$. In bad characteristic, this need no longer be true. Consider the example where $G$ is simple of type $E_{8}$ defined over a field of characteristic 2 . Let $C \in X_{G^{\prime}}$ be the unipotent class denoted by $D_{7}\left(a_{1}\right)$ in the table for $E_{8}$ in [20]. This class has non-trivial intersection with a Levi subgroup of type $D_{7}$ but, by [3, Proposition 3.6], it is a cuspidal class for $G^{\prime}$. On the other hand, the image of $C$ under $\pi_{G}$ still has non-trivial intersection with a Levi subgroup in $G$ of type $D_{7}$. But since $A_{G}(u)=1$ for $u \in \pi_{G}(C)$ (see the table in [20]), this now implies that $\pi_{G}(C)$ is non-cuspidal (in the sense of [3]).

\section{Some examples.}

Example 1. Let $G^{F}=\operatorname{Sp}_{4}(2)$. There are 6 unipotent characters; their degrees are given as follows (see [7, p.359]):

$\begin{array}{ccc}\text { polynomial degree } & \text { value at } q=2 & a_{\rho}, n_{\rho} \\ 1 & 1 & 0,1 \\ \frac{1}{2} q\left(q^{2}+1\right) & 5 & 1,2 \\ \frac{1}{2} q(q+1)^{2} & 9 & 1,2 \\ \frac{1}{2} q\left(q^{2}+1\right) & 5 & 1,2 \\ \frac{1}{2} q(q-1)^{2} & 1 & 1,2 \\ q^{4} & 16 & 4,1\end{array}$

The unique non-trivial character $\rho$ of degree 1 (with $a_{\rho}=1, n_{\rho}=2$ ) is a cuspidal unipotent character. There are 5 unipotent classes in the underlying algebraic group, with $\operatorname{dim} \mathcal{B}_{u}$ given by $4,2,2,1,0$, respectively (see [19, p.233]). Without looking at character values, it follows immediately from Theorem 3.7 that the unipotent support of $\rho$ must be the unique class with $\operatorname{dim} \mathcal{B}_{u}=1$. But it is not difficult to show (note that $G^{F} \cong \mathfrak{S}_{6}$ ) that $\rho$ has values \pm 1 on the two classes of regular unipotent elements in $G^{F}$ (the elements with $\operatorname{dim} \mathcal{B}_{u}=0$ ). 
This is the smallest example of a character which has a non-zero value on some element in a unipotent class of strictly bigger dimension than its unipotent support.

Example 2. Let $G^{F}=G_{2}(q)$ where $q \equiv-1 \bmod 3$. The underlying algebraic group has 5 unipotent classes, denoted by $1, A_{1}, \tilde{A}_{1}, G_{2}\left(a_{1}\right), G_{2}$, with $\operatorname{dim} \mathcal{B}_{u}$ given by $6,3,2,1,0$, respectively (see the table in [19, p.148]).

There is a unique family of unipotent characters of $G^{F}$ of size 8 , and all characters in this family have $a_{\rho}=1$ (see [7, p.372]). Using Theorem 3.7, it follows immediately that all characters in this family have unipotent support $G_{2}\left(a_{1}\right)$. In particular, the two cuspidal unipotent characters of $G^{F}$ have this class as their unipotent support.

Since $3 \mid q+1$, there exists a semisimple element $s \in G^{* F}$ (unique up to $G^{*}$ conjugacy) such that $C_{G^{*}}(s)^{F} \cong \mathrm{SU}_{3}(q)$. Since $\mathrm{SU}_{3}(q)$ has a cuspidal unipotent character of degree $q(q-1)$, the series of $G^{F}$ defined by $s$ contains a cuspidal character $\rho$ of degree $q\left(q^{3}-1\right)(q-1)$ (by the Jordan decomposition of characters). We have $a_{\rho}=1$ and $n_{\rho}=1$. Hence Theorem 3.7 implies that $G_{2}\left(a_{1}\right)$ is also the unipotent support of $\rho$.

This example shows that there can exist cuspidal irreducible characters which have the same unipotent support but which lie in series corresponding to semisimple elements $s, s^{\prime} \in T^{*}$ with $W_{s} \neq W_{s^{\prime}}$.

All of the remaining cuspidal characters lie in series corresponding to regular elements in the various anisotropic maximal tori of $G^{*}$. Their restriction to the unipotent elements is given by the Green function associated with the corresponding maximal torus. Hence the unipotent support of any such character is the class of regular unipotent elements.

Example 3. Let $G^{F}=G_{2}(q)$ where $q$ is a power of 3. Now $G$ has 6 unipotent classes, denoted by $1, A_{1},\left(\tilde{A}_{1}\right)_{3}, \tilde{A}_{1}, G_{2}\left(a_{1}\right), G_{2}$, with $\operatorname{dim} \mathcal{B}_{u}$ given by $6,3,3,2,1,0$, respectively (see the table in $[19$, p.148]). Let $\rho$ be a cuspidal unipotent character. As in Example 2, the unipotent support of $\rho$ is $G_{2}\left(a_{1}\right)$. We have $\left|A_{G}(u)\right|=2$ for $u \in G_{2}\left(a_{1}\right)$; hence the formula in Theorem 3.7 yields that $\operatorname{AV}\left(G_{2}\left(a_{1}\right), \rho\right)=$ $\pm 2 n_{\rho}^{-1} q^{d}$, where $d=\operatorname{dim} \mathcal{B}_{u}$ for $u \in G_{2}\left(a_{1}\right)$. From the table in [7, p.372] we see that $n_{\rho} \in\{2,3,6\}$. Hence, if $\rho$ is such that $n_{\rho}=3$ or 6 , then $n_{\rho}$ does not divide $\left|A_{G}(u)\right|$ (but still divides $2 q^{d}$, of course). Such a thing cannot happen in good characteristic, see [7, (13.4.3)].

Example 4. Let $G$ be simple modulo its center and of classical type $A_{n}, B_{n}, C_{n}$ or $D_{n}$. Assume that $q$ is odd and that $G^{F}$ has a cuspidal unipotent character $\rho_{0}$. Let $C_{0}$ be its unipotent support. By $[7,(8.5 .6)], \rho_{0}$ belongs to a (unique) $F$ stable cuspidal family of characters of $W$. From the explicit description of cuspidal families in $[7,(8.1)]$ and the form of the Fourier matrices for classical types in $[7$, Chap. 4] we conclude that $n_{\rho_{0}}=\left|A_{G}(u)\right|$ for $u \in C_{0}$ (cf. also [3, Theorem 3.3(c)]). Hence, using the formula in Theorem 3.7 we obtain that $\operatorname{AV}\left(C_{0}, \rho_{0}\right)= \pm q^{d}$, where $d=\operatorname{dim} \mathcal{B}_{u}$. In exceptional groups, it is not always true that the average value of a cuspidal unipotent character on its unipotent support is a power of $q$ (see, for example, type $G_{2}$ ).

\section{The Suzuki and Ree groups}

Now let $G$ be a simple group of type $B_{2}, G_{2}$ or $F_{4}$ defined over an algebraically closed field of characteristic $p=2,3,2$ respectively, and $F$ a non-standard Frobenius morphism such that the finite group of fixed points $G^{F}$ is a Suzuki or Ree group 
defined over a field $\mathbb{F}_{q^{2}}$ with $q^{2}$ an odd power of $p$. The irreducible characters of $G^{F}$ were determined by Suzuki, Ward and the second author respectively [15], [21], [22]. It turns out that the results of Lusztig on the classification of $\operatorname{Irr}\left(G^{F}\right)$ remain valid in these cases. More precisely, for each $\rho \in \operatorname{Irr}\left(G^{F}\right)$ there exist an $F$-stable conjugacy class of semisimple elements $s$ in the dual group and a $\gamma$-invariant family of irreducible characters of $W_{s}$. We write $E_{1}$ for the unique special character in the family of $\operatorname{Irr}\left(W_{s}\right)^{\gamma}$ attached to $\rho$. Then there exists an integer $n_{\rho} \geq 1$ such that the scalar product of $\rho$ with $R_{E_{1}}^{s}$ is given by $\pm n_{\rho}^{-1}$ (see $[7,14.2]$ for the case of $F_{4}$ ). It then turns out that the character degrees are all of the form

$$
\rho(1)= \pm n_{\rho}^{-1} \sqrt{p}^{-\epsilon} q^{a_{\rho}} N
$$

where $\epsilon=1$ if $a_{\rho}$ is odd and $\epsilon=0$ if $a_{\rho}$ is even, and where $N$ is an integer satisfying $N \equiv 1$ modulo $\sqrt{p} q$. Then the analogue of Theorem 3.7 holds:

Proposition 5.1. Assume that $G, p, F$ are as above. Let $\rho \in \operatorname{Irr}\left(G^{F}\right)$.

(1) There exists a unipotent support $C_{\rho}$ for $\rho$.

(2) Let $u \in C_{\rho}^{F}$ and $d=\operatorname{dim} \mathcal{B}_{u}$. Then we have

$$
\operatorname{AV}\left(C_{\rho}, \rho\right)= \pm n_{\rho}^{-1} \sqrt{p}^{-\epsilon} q^{d}\left|A_{G}(u)\right| \text { and } a_{\rho}=d,
$$

where $\epsilon=1$ if $a_{\rho}$ is odd and $\epsilon=0$ if $a_{\rho}$ is even.

Proof. This can be checked case by case from the known character tables (see [15], [21], [22]), the order relations on the sets of unipotent classes and the values of $d$ (see [19, Théorème II.10.15]).

We also obtain the following analogue of Corollary 4.2:

Corollary 5.2. Let $G, F$ be as above. Two unipotent characters of $G^{F}$ belong to the same family if and only if they have the same unipotent support.

Finally, we note that the assertion of Proposition 4.3 also remains true: it is sufficient to observe that the partial order on the set of $F$-stable unipotent classes is in fact a total order in these cases.

\section{REFERENCES}

[1] D.I. Deriziotis, On the number of conjugacy classes in finite groups of Lie type, Comm. in Algebra 13 (1985), 1019-1045. MR 86i:20067

[2] M. Geck, On the average values of the irreducible characters of finite groups of Lie type on geometric unipotent classes, Documenta Math. 1 (1996), 293-317. (electronic) MR 98c:20084

[3] M. Geck and G. Malle, Cuspidal unipotent classes and cuspidal Brauer characters, J. London Math. Soc. 53 (1996), 63-78. MR 97b:20013

[4] G. Laumon, Faisceaux caractères, Séminaire Bourbaki No. 709, Astérisque 177-178 (1989), 231-260. MR 91m:20062

[5] G. Lusztig, On the finiteness of the number of unipotent classes, Invent. Math. 34 (1976), 201-213. MR 54:7653

[6] G. Lusztig, Some problems in the representation theory of finite Chevalley groups, Proc. Symp. Pure Math., vol. 37, Amer. Math. Soc., Providence, 1980, pp. 313-317. MR 82i:20014

[7] G. Lusztig, Characters of reductive groups over a finite field, Ann. Math. Studies, vol. 107, Princeton U. Press, Princeton, 1984. MR 86j:20038

[8] G. Lusztig, Intersection cohomology complexes on a reductive group, Invent. Math. 75 (1984), 205-272. MR 86d:20050

[9] G. Lusztig, Character sheaves IV, Advances in Math. 59 (1986), 1-63. MR 87m:20118b

[10] G. Lusztig, Character sheaves V, Advances in Math. 61 (1986), 103-155. MR 87m:20118c 
[11] G. Lusztig, On the representations of reductive groups with disconnected centre, Orbites Unipotentes et Représentations, I. Groupes Finis et Algèbres de Hecke, Astérisque 168 (1988), 157-166. MR 90j:20083

[12] G. Lusztig, A unipotent support for irreducible representations, Advances in Math. 94 (1992), 139-179. MR 94a:20073

[13] G. Lusztig and N. Spaltenstein, Induced unipotent classes, J. London Math. Soc. 19 (1979), 41-52. MR 82g:20070

[14] G. Lusztig and N. Spaltenstein, On the generalized Springer correspondence for classical groups, Algebraic groups and related topics, Advanced Studies in Pure Math., vol. 6, Kinokuniya and North-Holland, Tokyo and Amsterdam, 1985, pp. 289-316. MR 87g:20072a

[15] G. Malle, Die unipotenten Charaktere von ${ }^{2} F_{4}\left(q^{2}\right)$, Comm. Algebra 18 (1990), 2361-2381. MR 91k:20015

[16] T. Shoji, Green functions of reductive groups over a finite field, Proc. Symp. Pure Math., vol. 47, Amer. Math. Soc., Providence, 1987, pp. 289-302. MR 88m:20014

[17] T. Shoji, Character sheaves and almost characters of reductive groups, II, Advances in Math. 111 (1995), 314-354. MR 95k:20069

[18] T. Shoji, Unipotent characters of finite classical groups, Finite Reductive Groups, Related Structures and Representations (M. Cabanes, ed.), Progress in Math., vol. 141, Birkhäuser, Boston, 1997, pp. 373-413. MR 98h:20079

[19] N. Spaltenstein, Classes unipotentes et sous-groupes de Borel, Lecture Notes in Math., vol. 946, Springer, Berlin Heidelberg New York, 1982. MR 84a:14024

[20] N. Spaltenstein, On the generalized Springer correspondence for exceptional groups, Algebraic groups and related topics, Advanced Studies in Pure Math., vol. 6, Kinokuniya and NorthHolland, Tokyo and Amsterdam, 1985, pp. 317-338. MR 87g:20072b

[21] M. Suzuki, On a class of doubly transitive groups, Ann. of Math. 75 (1962), 105-145. MR 25:112

[22] H.N. Ward, On Ree's series of simple groups, Trans. Amer. Math. Soc. 121 (1966), 62-89. MR 33:5752

U.F.R. De Mathématiques, Université Paris 7, et UMR 7586 du CNRS, 2 Place Jussieu, F-75251 PARIS Cedex 05, France

Current address: Institut Girard Desargues, Université Lyon 1, 69622 Villeurbanne Cedex, France

E-mail address: geck@desargues.univ-lyon1.fr

I.W.R., Im Neuenheimer Feld 368, D-69120 Heidelberg, Germany

Current address: FB Mathematik/Informatik, Heinrich-Plett-Str. 40, D-34132 Kassel, Germany

E-mail address: malle@mathematik.uni-kassel.de 